TRANSACTIONS OF THE

AMERICAN MATHEMATICAL SOCIETY

Volume 365, Number 5, May 2013, Pages 2241-2269

S 0002-9947(2012)05564-4

Article electronically published on September 18, 2012

\title{
ARCS ON DETERMINANTAL VARIETIES
}

\author{
ROI DOCAMPO
}

\begin{abstract}
We study arc spaces and jet schemes of generic determinantal varieties. Using the natural group action, we decompose the arc spaces into orbits, and analyze their structure. This allows us to compute the number of irreducible components of jet schemes, log canonical thresholds, and topological zeta functions.
\end{abstract}

\section{INTRODUCTION}

Let $\mathcal{M}=\mathbf{A}^{r s}$ denote the space of $r \times s$ matrices, and assume that $r \leq s$. Let $D^{k} \subset \mathcal{M}$ be the generic determinantal variety of rank $k$, that is, the subvariety of $\mathcal{M}$ whose points correspond to matrices of rank at most $k$. The purpose of this paper is to analyze the structure of arc spaces and jet schemes of generic determinantal varieties.

Arc spaces and jet schemes have attracted considerable attention in recent years. They were introduced to the field by J. F. Nash [Nas95, who noticed for the first time their connection with resolution of singularities. A few years later, M. Kontsevich introduced motivic integration [Kon95, DL99], popularizing the use of the arc space. Starting with the work of M. Mustaţă, arc and jets have become a standard tool in birational geometry, mainly because of their role in formulas for controlling discrepancies Mus01, Mus02, EMY03, ELM04, EM06, dFEI08.

But despite their significance from a theoretical point of view, arc spaces are often hard to compute in concrete examples. The interest in Nash's conjecture led to the study of arcs in isolated surface singularities LJ90, Nob91, LJR98, Plé05, PPP06, LJR08. Quotient singularities are analyzed from the point of view of motivic integration in DL02. We also understand the situation for monomial ideals GS06, Yue07b and for toric varieties Ish04. But beyond these cases very little is known about the geometry of the arc space of a singular algebraic variety. The purpose of this article is to analyze in detail the geometric structure of arc spaces and jet schemes of generic determinantal varieties, giving a new family of examples for which the arc space is well understood.

Recall that arcs and jets are higher order analogues of tangent vectors. Given a variety $X$ defined over $\mathbf{C}$, an $\operatorname{arc}$ of $X$ is a $\mathbf{C} \llbracket t \rrbracket$-valued point of $X$, and an $n$-jet is a $\mathbf{C}[t] /\left(t^{n+1}\right)$-valued point. A 1 -jet is the same as a tangent vector. Just as in the case of the tangent space, arcs on $X$ can be identified with the closed points of a scheme $X_{\infty}$, which we call the arc space of $X$ Nas95, Voj07. Similarly, $n$-jets give rise to the $n$-th jet scheme of $X$, which we denote by $X_{n}$ (see Section 1 for more details).

Received by the editors June 22, 2010 and, in revised form, February 7, 2011.

2010 Mathematics Subject Classification. Primary 14E18.

Key words and phrases. Arc spaces, jet schemes, determinantal varieties, topological zeta function.

(C)2012 American Mathematical Society 2241 
For the space of matrices $\mathcal{M}$, the arc space $\mathcal{M}_{\infty}$ and the jet scheme $\mathcal{M}_{n}$ can be understood set-theoretically as the spaces of matrices with entries in the rings $\mathbf{C} \llbracket t \rrbracket$ and $\mathbf{C}[t] /\left(t^{n+1}\right)$, respectively. $D_{\infty}^{k}$ and $D_{n}^{k}$ are contained in $\mathcal{M}_{\infty}$ and $\mathcal{M}_{n}$, and their equations are obtained by "differentiating" the $k \times k$ minors of a matrix of independent variables. We approach the study of $D_{\infty}^{k}$ and $D_{n}^{k}$ with three goals in mind: understand the topology of $D_{n}^{k}$, compute log canonical thresholds for the pairs $\left(\mathcal{M}, D^{k}\right)$, and compute topological zeta functions for $\left(\mathcal{M}, D^{k}\right)$.

0.1 . Irreducible components of jet schemes. The topology of the jet scheme $D_{n}^{k}$ is intimately related to the generalized Nash problem.

Given an irreducible family of $\operatorname{arcs} \mathcal{C} \subset \mathcal{M}_{\infty}$, we can consider $\nu_{\mathcal{C}}$, the order of vanishing along a general element of $\mathcal{C}$. The function $\nu_{\mathcal{C}}$ is almost a discrete valuation, the only problem being that it takes infinite value on those functions vanishing along all the $\operatorname{arcs}$ in $\mathcal{C}$. If there are no such functions, we call the family fat [sh08, and we see that irreducible fat families of arcs give rise to discrete valuations.

Conversely, given a divisorial valuation $\nu$ over $\mathcal{M}$, any isomorphism from $\mathbf{C} \llbracket t \rrbracket$ to the completion of the valuation ring produces a non-closed point of $\mathcal{M}_{\infty}$. The closure of any of these points can be easily seen to give an irreducible fat family of arcs inducing $\nu$.

Among all closed irreducible fat families of arcs inducing a given divisorial valuation, there exists a maximal one with respect to the order of containment, known as the maximal divisorial set (see Section 1 for details). In this way we get a bijection between divisorial valuations and maximal divisorial sets in the arc space, and we can use the topology in the arc space to give structure to the set of divisorial valuations. More concretely, the containment of maximal divisorial sets induces a partial order on valuations. The understanding of this order is known as the generalized Nash problem Ish08.

There are other ways to define orders in the set of divisorial valuations. For example, thinking of valuations as functions on $\mathcal{O}_{\mathcal{M}}$, we can partially order them by comparing their values. In dimension two, the resolution process also gives an order. It can be shown that the order induced by the arc space is different from any previously known order Ish08, but beyond that, not much is known about the generalized Nash problem. A notable exception is the case of toric valuations on toric varieties, which was studied in detail in Ish04.

Determining the irreducible components for $D_{n}^{k}$ is essentially equivalent to computing minimal elements among those valuations over $\mathcal{M}$ that satisfy certain contact conditions with respect to $D^{k}$. In Section 4 we solve the generalized Nash problem for invariant divisorial valuations, and we use this to prove the following theorem.

Theorem A. Let $D^{k}$ be the determinantal variety of matrices of size $r \times s$ and rank at most $k$, where $k<r \leq s$. Let $D_{n}^{k}$ be the $n$-th jet scheme of $D^{k}$. If $k=0$ or $k=r-1$, the jet scheme $D_{n}^{k}$ is irreducible. Otherwise the number of irreducible components of $D_{n}^{k}$ is

$$
n+2-\left\lceil\frac{n+1}{k+1}\right\rceil .
$$


Jet schemes for determinantal varieties were previously studied in KS05a, KS05b Yue07a. Up to now, the approach has always been to use techniques from commutative algebra, performing a careful study of the defining equations. This has been quite successful for ranks 1 and $r-1$, especially for square matrices, but the general case seems too complex for these methods.

Our approach is quite different in nature: we focus on the natural group action. This is a technique that already plays a central role in Ishii's study of the arc spaces of toric varieties Ish04. Consider the group $G=\mathrm{GL}_{r} \times \mathrm{GL}_{s}$, which acts on the space of matrices $\mathcal{M}$ via a change of basis. The rank of a matrix is the unique invariant for this action, the orbit closures being precisely the determinantal varieties $D^{k}$. The assignments sending a variety $X$ to its arc space $X_{\infty}$ and its jet schemes $X_{n}$ are functorial. Since $G$ is an algebraic group and its action on $\mathcal{M}$ is rational, we see that $G_{\infty}$ and $G_{n}$ are also groups, and that they act on $\mathcal{M}_{\infty}$ and $\mathcal{M}_{n}$, respectively. Determinantal varieties are $G$-invariant, hence their arc spaces are $G_{\infty}$-invariant and their jet schemes are $G_{n}$-invariant. The main observation is that most questions regarding components and dimensions of jet schemes and arc spaces of determinantal varieties can be reduced to the study of orbits in $\mathcal{M}_{\infty}$ and $\mathcal{M}_{n}$.

Orbits in the arc space $\mathcal{M}_{\infty}$ are easy to classify. As a set, $\mathcal{M}_{\infty}$ is just the space of matrices with coefficients in $\mathbf{C} \llbracket t \rrbracket$, and $G_{\infty}$ acts via a change of basis over the ring $\mathbf{C} \llbracket t \rrbracket$. Gaussian elimination allows us to find representatives for the orbits: each of them contains a unique diagonal matrix of the form $\operatorname{diag}\left(t^{\lambda_{1}}, \ldots, t^{\lambda_{r}}\right)$, where $\infty \geq \lambda_{1} \geq \cdots \geq \lambda_{r} \geq 0$, and the sequence $\lambda=\left(\lambda_{1}, \ldots, \lambda_{r}\right)$ determines the orbit. In Section 3 we see how to decompose arc spaces and jet schemes of determinantal varieties as unions of these orbits. Once this is done, the main difficulty in determining irreducible components is the understanding of the generalized Nash problem for orbits closures in $\mathcal{M}_{\infty}$. This is the purpose of the following theorem, which is proven in the present article as Theorem 4.7.

Theorem B (Nash problem for invariant valuations). Consider two sequences $\lambda=$ $\left(\lambda_{1} \geq \cdots \geq \lambda_{r} \geq 0\right)$ and $\lambda^{\prime}=\left(\lambda_{1}^{\prime} \geq \cdots \geq \lambda_{r}^{\prime} \geq 0\right)$, and let $\mathcal{C}_{\lambda}$ and $\mathcal{C}_{\lambda^{\prime}}$ be the corresponding orbits in the arc space $\mathcal{M}_{\infty}$. Then the closure of $\mathcal{C}_{\lambda}$ contains $\mathcal{C}_{\lambda^{\prime}}$ if and only if

$$
\lambda_{r}+\lambda_{r-1}+\cdots+\lambda_{r-k} \leq \lambda_{r}^{\prime}+\lambda_{r-1}^{\prime}+\cdots+\lambda_{r-k}^{\prime} \quad \forall k \in\{0, \ldots, r\} .
$$

Sequences of the form $\left(\infty \geq \lambda_{1} \geq \lambda_{2} \geq \cdots \geq \lambda_{r} \geq 0\right)$ are closely related to partitions (the only difference being the possible presence of infinite terms), and the order that appears in the above theorem is a modification of a well-known order on the set of partitions: the order of domination (see Section 2). Since the poset of partitions is well understood, one has very explicit information about the structure of the poset of orbits in the arc space. This allows us to compute minimal elements among some interesting families of orbits, leading to the proof of Theorem A (see Section 44).

0.2. Log canonical thresholds. Mustată's formula Mus01, ELM04, dFEI08, allows us to compute log discrepancies for divisorial valuations by computing codimensions of the appropriate sets in the arc space. In the case at hand, the most natural valuations one can look at are the invariant divisorial valuations. In Section 5 we see that the maximal divisorial sets corresponding to these valuations are precisely the orbit closures in $\mathcal{M}$. Hence computing log discrepancies gets reduced 
to computing codimensions of orbits. This explains the relevance of the following result, which appears in Section 5 as Proposition 5.4.

Theorem C (Log discrepancies of invariant valuations). Consider a sequence $\lambda=$ $\left(\lambda_{1} \geq \cdots \geq \lambda_{r} \geq 0\right)$ and let $\mathcal{C}_{\lambda}$ be the corresponding orbit in the arc space $\mathcal{M}_{\infty}$. Then the codimension of $\mathcal{C}_{\lambda}$ in $\mathcal{M}_{\infty}$ is

$$
\operatorname{codim}\left(\mathcal{C}_{\lambda}, \mathcal{M}_{\infty}\right)=\sum_{i=1}^{r} \lambda_{i}(s-r+2 i-1) .
$$

Once these codimensions are known, one can compute log canonical thresholds for pairs involving determinantal varieties. The following result appears in Section 5 as Theorem 5.6.

Theorem D. Let $\mathcal{M}$ be the space of matrices of size $r \times s$, and $D^{k}$ the subvariety of $\mathcal{M}$ containing matrices of rank at most $k$. The log canonical threshold of the pair $\left(\mathcal{M}, D^{k}\right)$ is

$$
\operatorname{lct}\left(\mathcal{M}, D^{k}\right)=\min _{i=0, \ldots, k} \frac{(r-i)(s-i)}{k+1-i} .
$$

We should note that the previous result is not new. Log resolutions for generic determinantal varieties are now classical objects. They are essentially spaces of complete collineations, obtained by blowing up $D^{k}$ along $D^{0}, D^{1}, \ldots, D^{k-1}$, in this order Sem51, Tyr56, Vai84, Lak87. It is possible to use these resolutions to compute log canonical thresholds, and this was done by A. Johnson in her Ph.D. thesis [Joh03. In fact she is able to compute all the multiplier ideals $\mathcal{J}\left(\mathcal{M}, c \cdot D^{k}\right)$. Our method does not need any knowledge about the structure of these $\log$ resolutions.

0.3. Topological zeta function. Using our techniques, we are able to understand orbits in $\mathcal{M}_{\infty}$ quite explicitly. In Section 6 we compute motivic volumes of orbits, and this allows us to determine topological zeta functions for determinantal varieties (for square matrices).

Theorem E. Let $\mathcal{M}=\mathbf{A}^{r^{2}}$ be the space of square $r \times r$ matrices, and let $D^{k}$ be the subvariety of matrices of rank at most $k$. Then the topological zeta function of the pair $\left(\mathcal{M}, D^{k}\right)$ is given by

$$
Z_{D^{k}}^{\mathrm{top}}(s)=\prod_{\zeta \in \Omega} \frac{1}{1-s \zeta^{-1}}
$$

where $\Omega$ is the set of poles:

$$
\Omega=\left\{-\frac{r^{2}}{k+1}, \quad-\frac{(r-1)^{2}}{k}, \quad-\frac{(r-2)^{2}}{k-1}, \quad \ldots, \quad-(r-k)^{2}\right\} .
$$

\section{Ard spaces and motivic integration}

In this section we briefly review the basic theory of arc spaces and motivic integration, as these tools will be used repeatedly. Most of these results are well known. We have gathered them mainly from DL98, DL99, [ELM04, Ish08, Vey06, and dFEI08. We direct the reader to those papers for more details and proofs. 
We will always work with varieties and schemes defined over the complex numbers. When we use the word scheme, we do not necessarily assume that it is of finite type.

1.1. Arcs and jets. Given a variety $X$ and a non-negative integer $n$, we consider the following functor from the category of $\mathbf{C}$-algebras to the category of sets:

$$
F_{X}^{n}(A)=\operatorname{Hom}\left(\operatorname{Spec} A[t] /\left(t^{n+1}\right), X\right) .
$$

This functor is representable by a scheme, the $n$-th jet scheme of $X$, which we denote by $X_{n}$. By construction, when $m \geq n$ we have natural projections $\psi_{m, n}$ : $X_{m} \rightarrow X_{n}$, known as the truncation maps. The inverse limit of the jet schemes of $X$ (with respect to the truncation maps) is again a scheme, known as the arc space of $X$, and denoted by $X_{\infty}$ :

$$
X_{\infty}=\lim _{n} X_{n}
$$

Notice that $X_{\infty}$ is not of finite type if $\operatorname{dim} X>0$, and that we have natural projections $\psi_{n}: X_{\infty} \rightarrow X_{n}$, also known as truncation maps. When $X$ is affine, the arc space represents the following functor:

$$
F_{X}^{\infty}(A)=\operatorname{Hom}(\operatorname{Spec} A \llbracket t \rrbracket, X) .
$$

The assignment $X \mapsto X_{\infty}$ is functorial: each morphism $f: X^{\prime} \rightarrow X$ induces by composition a morphism $f_{\infty}: X_{\infty}^{\prime} \rightarrow X_{\infty}$, and $(g \circ f)_{\infty}=f_{\infty} \circ g_{\infty}$. As a consequence, if $G$ is a group scheme, so is $G_{\infty}$, and if $X$ has an action by $G$, the arc space $X_{\infty}$ has an action by $G_{\infty}$. Analogous statements hold for the jet schemes.

1.2. Contact loci and valuations. By a constructible subset of a scheme (not necessarily Noetherian) we mean a finite union of locally closed subsets. A constructible subset $\mathcal{C} \subset X_{\infty}$ is called thin if one can find a proper subscheme $Y \subset X$ such that $\mathcal{C} \subset Y_{\infty}$. Constructible subsets which are not thin are called fat. A cylinder in $X_{\infty}$ is a set of the form $\psi_{n}^{-1}(C)$ for some constructible set $C \subset X_{n}$. On a smooth variety, cylinders are fat, but in general a cylinder might be contained in $S_{\infty}$, where $S=\operatorname{Sing}(X) \subset X$ is the singular locus.

An $\operatorname{arc} \alpha \in X_{\infty}$ induces a morphism $\alpha:$ Spec $K \llbracket t \rrbracket \rightarrow X$, where $K$ is the residue field of $\alpha$. Given an ideal $\mathcal{I} \subset \mathcal{O}_{X}$, its pull-back $\alpha^{*}(\mathcal{I}) \subset K \llbracket t \rrbracket$ is of the form $\left(t^{e}\right)$, where $e$ is either a non-negative integer or infinity (by convention $t^{\infty}=0$ ). We call $e$ the order of contact of $\alpha$ along $\mathcal{I}$ and denote it by $\operatorname{ord}_{\alpha}(\mathcal{I})$. Given a collection of ideals $I=\left(\mathcal{I}_{1}, \ldots, \mathcal{I}_{r}\right)$ and a multi-index $\mu=\left(m_{1}, \ldots, m_{r}\right) \in \mathbf{Z}_{\geq 0}$, we introduce the contact locus:

$$
\begin{gathered}
\operatorname{Cont}^{=\mu}(I)=\left\{\alpha \in X_{\infty}: \operatorname{ord}_{\alpha}\left(\mathcal{I}_{j}\right)=m_{j} \text { for all } j\right\}, \\
\operatorname{Cont}^{\mu}(I)=\left\{\alpha \in X_{\infty}: \operatorname{ord}_{\alpha}\left(\mathcal{I}_{j}\right) \geq m_{j} \text { for all } j\right\} .
\end{gathered}
$$

Notice that contact loci are cylinders.

Let $\mathcal{C} \subset X_{\infty}$ be an irreducible fat set. Then $\mathcal{C}$ contains a generic point $\gamma \in \mathcal{C}$ which we interpret as a morphism $\gamma: \operatorname{Spec} K \llbracket t \rrbracket \rightarrow X$, where $K$ is the residue field of $\gamma$. Let $\eta$ be the generic point of Spec $K \llbracket t \rrbracket$. Since $\mathcal{C}$ is fat, $\gamma(\eta)$ is the generic point of $X$, and we get an inclusion of fields,

$$
\mathbf{C}(X) \rightarrow K((t))
$$


The composition of this inclusion with the canonical valuation on $K((t))$ is a valuation on $\mathbf{C}(X)$, which we denote by $\nu_{\mathcal{C}}$. In this way we obtain a map from the set of fat irreducible subsets of $X_{\infty}$ to the set of valuation of $\mathbf{C}(X)$ defined over $X$ :

$\left\{\mathcal{C} \subseteq X_{\infty}: \mathcal{C}\right.$ irreducible fat $\} \quad \longrightarrow \quad\{$ discrete valuations over $X\}$.

This map is always surjective: for a discrete valuation $\nu$ of $\mathbf{C}(X)$ defined over $X$, the completion of the discrete valuation ring $\mathcal{O}_{\nu}$ is isomorphic to a power series ring $k_{\nu} \llbracket t \rrbracket$. But it is far from being injective. For example, different choices of a uniformizing parameter in a discrete valuation ring give rise to different arcs.

A valuation $\nu$ of $\mathbf{C}(X)$ is called divisorial if it is of the form $q \cdot \operatorname{val}_{E}$, where $q$ is a positive integer and $E$ is a prime divisor on a variety $X^{\prime}$ birational to $X$. An irreducible fat set $\mathcal{C} \subset X_{\infty}$ is said to be divisorial if the corresponding valuation $\nu_{\mathcal{C}}$ is divisorial. In Ish08 it is shown that the union of all divisorial sets corresponding to a given valuation $\nu$ is itself a divisorial set defining $\nu$ (in fact it is an irreducible component of a contact locus). These unions are called maximal divisorial sets. There is a one-to-one correspondence between divisorial valuations and maximal divisorial sets. This gives an inclusion

$$
\{\text { divisorial valuations over } X\} \hookrightarrow\left\{\mathcal{C} \subseteq X_{\infty}: \mathcal{C} \text { irreducible fat }\right\} \text {. }
$$

Through this inclusion, the topology on the arc space $X_{\infty}$ gives structure to the set of divisorial valuations. For example, given two valuations $\nu$ and $\nu^{\prime}$ with corresponding maximal divisorial sets $\mathcal{C}$ and $\mathcal{C}^{\prime}$, we say that $\nu$ dominates $\nu^{\prime}$ if $\mathcal{C} \supseteq \mathcal{C}^{\prime}$. The generalized Nash problem consists in understanding the relation of domination among divisorial valuations.

1.3. Discrepancies. Let $X$ be a variety of dimension $n$. The Nash blowing-up of $X$, denoted $\widehat{X}$, is defined as the closure of $X_{\text {reg }}$ in $\mathbf{P}_{X}\left(\Omega_{X}^{n}\right)$. It is equipped with a tautological line bundle $\left.\mathcal{O}_{\mathbf{P}_{X}\left(\Omega_{X}^{n}\right)}(1)\right|_{\widehat{X}}$, which we denote by $\widehat{K}_{X}$ and call the Mather canonical line bundle of $X$. When $X$ is smooth, $X=\widehat{X}$ and $K_{X}=\widehat{K}_{X}$.

When $Y$ is a smooth variety and $f: Y \rightarrow X$ is a birational morphism that factors through the Nash blowing-up, we define the relative Mather canonical divisor of $f$ as the unique effective divisor supported on the exceptional locus of $f$ and linearly equivalent to $K_{Y}-f^{*} \widehat{K}_{X}$. We denote it by $\widehat{K}_{Y / X}$.

Let $\nu$ be a divisorial valuation of $X$. Then we can find a smooth variety $Y$ and a birational map $Y \rightarrow X$ factoring through the Nash blowing-up of $X$, such that $\nu=q \cdot \operatorname{val}_{E}$ for some prime divisor $E \subset Y$. We define the Mather discrepancy of $X$ along $\nu$ as

$$
\hat{k}_{\nu}(X)=q \cdot \operatorname{ord}_{E}\left(\widehat{K}_{Y / X}\right) .
$$

This definition is independent of the choice of resolution $Y$.

In the smooth case, Mustaţă showed that we can compute discrepancies using the arc space [Mus01, ELM04]. This is generalized to arbitrary varieties in dFEI08. via the use of Mather discrepancies. More precisely, given a divisorial valuation $\nu=q \cdot \operatorname{val}_{E}$, let $\mathcal{C}_{\nu} \subset X_{\infty}$ be the corresponding maximal divisorial set. Then

$$
\operatorname{codim}\left(\mathcal{C}_{\nu}, X_{\infty}\right)=\hat{k}_{\nu}(X)+q .
$$

1.4. Motivic integration. Let $\mathcal{M}_{0}$ be the Grothendieck ring of algebraic varieties over C. In DL99, the authors introduce a certain completion of a localization of $\mathcal{M}_{0}$, which we denote by $\mathcal{M}$. Also, for each variety $X$ over $\mathbf{C}$, they define a measure 
$\mu_{X}$ on $X_{\infty}$ with values in $\mathcal{M}$. This measure is known as the motivic measure of $X$. The following properties hold for $\mathcal{M}$ and the measures $\mu_{X}$ :

- There is a canonical ring homomorphism $\mathcal{M}_{0} \rightarrow \mathcal{M}$. In particular, for each variety $X$ one can associate an element $[X] \in \mathcal{M}$, and the map $X \mapsto[X]$ is additive (meaning that $[X]=[Y]+[U]$, where $Y \subset X$ is a closed subvariety and $U=X \backslash Y)$.

- The element $\left[\mathbf{A}^{1}\right] \in \mathcal{M}$ has a multiplicative inverse. We write $\mathbf{L}=\left[\mathbf{A}^{1}\right]$.

- Both the Euler characteristic and the Hodge-Deligne polynomial, considered as ring homomorphisms with domain $\mathcal{M}_{0}$, extend to homomorphisms

$$
\chi: \mathcal{M} \rightarrow \mathbf{R}, \quad E: \mathcal{M} \rightarrow \mathbf{Z}((u, v)),
$$

where $\chi(\mathbf{L})=1$ and $E(\mathbf{L})=u v$.

- Cylinders in $X_{\infty}$ are $\mu_{X}$-measurable. In particular, contact loci are measurable.

- If $X$ is smooth, $\mu_{X}\left(X_{\infty}\right)=[X]$.

- A thin measurable set has measure zero.

- Let $\mathcal{C} \subset X_{\infty}$ be a cylinder in $X_{\infty}$. Then the truncations $\psi_{n}(\mathcal{C}) \subset X_{n}$ are of finite type, so they define elements $\left[\psi_{n}(\mathcal{C})\right] \in \mathcal{M}$. Then

$$
\mu_{X}(\mathcal{C})=\lim _{n \rightarrow \infty}\left[\psi_{n}(\mathcal{C})\right] \cdot \mathbf{L}^{-n d},
$$

where $d$ is the dimension of $X$. Furthermore, if $\mathcal{C}$ does not intersect $\left(X_{\text {sing }}\right)_{\infty}$, then $\left[\psi_{n}(\mathcal{C})\right] \cdot \mathbf{L}^{-n d}$ stabilizes for $n$ large enough.

- Given an ideal $\mathcal{I} \subset \mathcal{O}_{X}$, we define a function $|\mathcal{I}|$ on $X_{\infty}$ with values on $\mathcal{M}$ via

$$
|\mathcal{I}|(\alpha)=\mathbf{L}^{-\operatorname{ord}_{\alpha}(\mathcal{I})}, \quad \alpha \in X_{\infty} .
$$

Notice that $\operatorname{ord}_{\beta}(\mathcal{I})=\infty$ if and only if $\beta \in \operatorname{Zeroes}(\mathcal{I})_{\infty}$, so $|\mathcal{I}|$ is only defined up to a measure zero set. Then $|\mathcal{I}|$ is $\mu_{X}$-integrable and

$$
\int_{X_{\infty}}|\mathcal{I}| d \mu_{X}=\sum_{p=0}^{\infty} \mu_{X}\left(\operatorname{Cont}^{=p}(\mathcal{I})\right) \cdot \mathbf{L}^{-p} .
$$

- Let $f: Y \rightarrow X$ be a birational map, and assume $Y$ smooth. Let $\operatorname{Jac}(f)$ be the ideal in $\mathcal{O}_{Y}$ for which $f^{*} \Omega_{X}^{n} \rightarrow \operatorname{Jac}(f) \cdot \Omega_{Y}^{n}$ is surjective. Then $\left(f_{\infty}\right)^{*}\left(\mu_{X}\right)=|\operatorname{Jac}(f)| \cdot \mu_{Y}$. In other words, for a measurable set $\mathcal{C} \subset X_{\infty}$, and a $\mu_{X}$-integrable function $\varphi$,

$$
\int_{\mathcal{C}} \varphi d \mu_{X}=\int_{f_{\infty}^{-1}(\mathcal{C})}\left(\varphi \circ f_{\infty}\right)|\operatorname{Jac}(f)| d \mu_{Y}
$$

This is known as the change of variables formula for motivic integration.

- Assume that $X$ is smooth, and consider a subscheme $Y \subset X$ with ideal $\mathcal{I} \subset \mathcal{O}_{X}$. The motivic Igusa zeta function of the pair $(X, Y)$ is defined as

$$
Z_{Y}(s)=\int_{X_{\infty}}|\mathcal{I}|^{s} d \mu_{X}=\sum_{p=0}^{\infty} \mu_{X}\left(\operatorname{Cont}^{=p}(\mathcal{I})\right) \cdot \mathbf{L}^{-s p} .
$$

In this expression, $\mathbf{L}^{-s}$ is to be understood as a formal variable, so $Z_{Y}(s) \in$ $\mathcal{M} \llbracket \mathbf{L}^{-s} \rrbracket$. It is shown in DL98 that $Z_{Y}(s)$ is a rational function. More precisely, let $f: X^{\prime} \rightarrow X$ be a $\log$ resolution of the pair $(X, Y)$. This means that $f$ is a proper birational map, $X^{\prime}$ is smooth, the scheme-theoretic inverse image of $Y$ in $X^{\prime}$ is an effective Cartier divisor $E$, the map $f$ is an 
isomorphism on $X^{\prime} \backslash E$, and the divisor $K_{X^{\prime} / X}+E$ has simple normal crossings. Let $E_{j}, j \in J$, be the irreducible components of $E$, and write $E=\sum_{j \in J} a_{j} E_{j}$ and $K_{X^{\prime} / X}=\sum_{j \in J} k_{j} E_{j}$. For a subset $I \subset J$, consider $E_{I}^{\circ}=\left(\bigcap_{i \in I} E_{i}\right) \backslash\left(\bigcup_{j \notin I} E_{j}\right)$. Then:

$$
Z_{Y}(s)=\sum_{I \subseteq J}\left[E_{I}^{\circ}\right] \prod_{i \in I} \frac{\mathbf{L}^{-a_{i} s}(\mathbf{L}-1)}{\mathbf{L}^{k_{i}+1}-\mathbf{L}^{-a_{i} s}} .
$$

- Keeping the same notation as above, the topological zeta function of the pair $(X, Y)$ is defined as

$$
Z_{Y}^{\mathrm{top}}(s)=\sum_{I \subseteq J} \chi\left(E_{I}^{\circ}\right) \prod_{i \in I} \frac{1}{a_{i} s+k_{i}+1},
$$

where $\chi(\cdot)$ denotes the Euler characteristic (see Vey06, DL98]). Notice that when $s$ is a positive integer we have

$$
\frac{\mathbf{L}^{-a_{i} s}(\mathbf{L}-1)}{\mathbf{L}^{k_{i}+1}-\mathbf{L}^{-a_{i} s}}=\frac{1}{1+\mathbf{L}+\mathbf{L}^{2}+\cdots+\mathbf{L}^{a_{i} s+k_{i}}}=\frac{1}{\left[\mathbf{P}^{a_{i} s+k_{i}}\right]},
$$

and that the Euler characteristic of $\mathbf{P}^{d}$ is $d+1$. In this sense, the topological zeta function can be understood as the specialization of the motivic Igusa zeta function via the Euler characteristic map $\chi: \mathcal{M} \rightarrow \mathbf{R}$. One can use this fact to show that $Z_{Y}^{\text {top }}(s)$ is independent of the chosen log resolution DL98.

- As explained in DL98, §2.3] one can also understand the relation between $Z_{Y}(s)$ and $Z_{Y}^{\text {top }}(s)$ in the following way. Let $\mathcal{A}$ be the subring of $\mathcal{M} \llbracket \mathbf{L}^{-s} \rrbracket$ generated by the polynomials in $\mathcal{M}\left[\mathbf{L}^{-s}\right]$ and by the quotients of the form $\frac{\mathbf{L}^{-a s}(\mathbf{L}-1)}{\mathbf{L}^{k+1}-\mathbf{L}^{-a s}}$ for positive integers $a, k$. The expansions

$$
\begin{aligned}
& \mathbf{L}^{-s}=\sum_{n=0}^{\infty}\left(\begin{array}{c}
-s \\
n
\end{array}\right)(\mathbf{L}-1)^{n}=1-s(\mathbf{L}-1)+\frac{-s(-s-1)}{2}(\mathbf{L}-1)^{2}+\ldots, \\
& \frac{\mathbf{L}^{-a s}(\mathbf{L}-1)}{\mathbf{L}^{k+1}-\mathbf{L}^{-a s}}=\frac{1}{1+\mathbf{L}+\cdots+\mathbf{L}^{a s+k}}=\frac{1}{a s+k+1}-\frac{a s+k}{2(a s+k+1)}(\mathbf{L}-1)+\ldots
\end{aligned}
$$

induce a map from $\mathcal{A}$ to $\overline{\mathcal{M}}(s) \llbracket \mathbf{L}-1 \rrbracket$, where $\overline{\mathcal{M}}$ is the largest quotient of $\mathcal{M}$ with no $(\mathbf{L}-1)$-torsion. Using the Euler characteristic map $\chi: \mathcal{M} \rightarrow \mathbf{R}$ (which sends $\mathbf{L}$ to 1 and factors through $\overline{\mathcal{M}}$ ) and considering the quotient by the ideal generated by $(\mathbf{L}-1)$, we get a natural map $\operatorname{ev}_{\mathbf{L}=1}: \mathcal{A} \rightarrow \mathbf{R}(s)$. The motivic Igusa zeta function is an element of $\mathcal{A}$, and the topological zeta function is its image in $\mathbf{R}(s)$ via $\operatorname{ev}_{\mathbf{L}=1}$ :

$$
Z_{Y}^{\text {top }}(s)=\operatorname{ev}_{\mathbf{L}=1}\left(Z_{Y}(s)\right) .
$$

\section{Partitions}

In order to enumerate orbits in the arc space of determinantal varieties, it will be convenient to use the language of partitions. In fact, we will consider a slight generalization of the concept of partition, where we allow terms of infinite size and an infinite number of terms (we call these objects pre-partitions). In this section we recall some basic facts about partitions that will be needed in the rest of this 
article, and extend them to the case of pre-partitions. Most of the results are well known. For a detailed account of the theory of partitions we refer the reader to dCEP80, and [Ful97.

2.1. Definitions. Let $\mathbf{N}$ denote the set of non-negative integers, and consider $\overline{\mathbf{N}}=$ $\mathbf{N} \cup\{\infty\}$. We extend the natural order on $\mathbf{N}$ to $\overline{\mathbf{N}}$ by setting $\infty>n$ for any $n \in \mathbf{N}$. We also set $\infty+n=\infty$ for any $n \in \overline{\mathbf{N}}$.

A pre-partition is an infinite non-increasing sequence of elements of $\overline{\mathbf{N}}$. Given a pre-partition $\lambda=\left(\lambda_{1}, \lambda_{2}, \ldots\right)$, the elements $\lambda_{i}$ are known as the terms of $\lambda$. The first term $\lambda_{1}$ is called the maximal term or co-length of $\lambda$. If all the terms of $\lambda$ are non-zero, we say that $\lambda$ has infinite length; otherwise, the largest integer $i$ such that $\lambda_{i} \neq 0$ is called the length of $\lambda$. If a pre-partition $\lambda$ has length no larger than $\ell$, we will often denote $\lambda$ by the finite sequence $\left(\lambda_{1}, \lambda_{2}, \ldots, \lambda_{\ell}\right)$.

A partition is a finite non-increasing sequence of positive integers. A partition can be naturally identified with a pre-partition of finite length and finite co-length.

Given a pre-partition $\lambda=\left(\lambda_{1}, \lambda_{2}, \ldots\right)$ we define

$$
\lambda_{i}^{*}=\sup \left\{j: \lambda_{j} \geq i\right\} \in \overline{\mathbf{N}} \text {. }
$$

Then $\lambda_{i}^{*} \geq \lambda_{i+1}^{*}$, and we obtain a new pre-partition $\lambda^{*}$, known as the conjugate pre-partition of $\lambda$. It follows from the definition that $\lambda^{* *}=\lambda$, that the length of $\lambda^{*}$ is the co-length of $\lambda$, and that the co-length of $\lambda^{*}$ is the length of $\lambda$. In particular, the conjugate of a partition is also a partition.

2.2. Diagrams. It will be helpful to visualize pre-partitions as Young diagrams (sometimes also known as Ferrers diagrams). A Young diagram is a graphical representation of a pre-partition; it is a collection of boxes, arranged in left-justified rows, with non-increasing row sizes. To each pre-partition $\lambda=\left(\lambda_{1}, \lambda_{2}, \ldots\right)$ there is a unique Young diagram whose $i$-th row has size $\lambda_{i}$. For example:
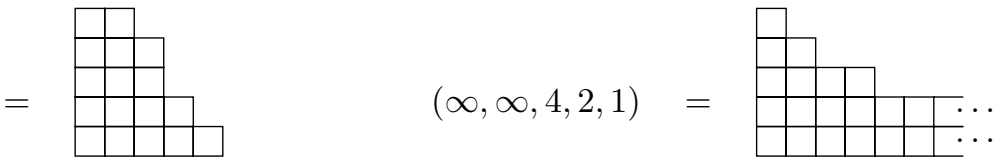

The length of a partition corresponds to the height of the associated diagram, whereas the co-length corresponds to the width. The diagram of the conjugate prepartition is obtained from the original diagram by switching rows with columns. More concretely, if $T$ denotes the diagram associated to a pre-partition $\lambda$, the terms $\lambda_{i}$ of the pre-partition give the row sizes of $T$, and the terms $\lambda_{i}^{*}$ of the conjugate pre-partition give the column sizes of $T$.

2.3. Posets of partitions. Given two pre-partitions $\lambda=\left(\lambda_{1}, \lambda_{2}, \ldots\right)$ and $\mu=$ $\left(\mu_{1}, \mu_{2}, \ldots\right)$, we say that $\lambda$ is contained in $\mu$, and denote it by $\lambda \subseteq \mu$, if $\lambda_{i} \leq \mu_{i}$ for all $i$. Containment of pre-partitions corresponds to containment of the associated diagrams. In particular, $\lambda \subseteq \mu$ if and only if $\lambda^{*} \subseteq \mu^{*}$.

If $\lambda$ and $\mu$ are pre-partitions with finite co-length, we say that $\mu$ dominates $\lambda$, denoted by $\lambda \leq \mu$, if

$$
\lambda_{1}+\lambda_{2}+\cdots+\lambda_{i} \leq \mu_{1}+\mu_{2}+\cdots+\mu_{i}
$$

for all positive integers $i$. If $\lambda$ and $\mu$ have finite length, we say that $\mu$ co-dominates $\lambda$, denoted $\lambda \triangleleft \mu$, if

$$
\lambda_{i}+\lambda_{i+1}+\ldots \leq \mu_{i}+\mu_{i+1}+\ldots
$$


for all positive integers $i$ (notice that the sums above have only a finite number of terms because the pre-partitions have finite length). It is shown in dCEP80, Prop. 1.1] that the conditions of domination and co-domination of pre-partitions can be expressed in terms of the conjugates. More precisely, we have:

$$
\begin{aligned}
& \lambda \leq \mu \Longleftrightarrow \lambda^{*} \triangleleft \mu^{*} \Longleftrightarrow \lambda_{i}^{*}+\lambda_{i+1}^{*}+\cdots \leq \mu_{i}^{*}+\mu_{i+1}^{*}+\ldots \quad \forall i, \\
& \lambda \triangleleft \mu \Longleftrightarrow \lambda^{*} \leq \mu^{*} \Longleftrightarrow \lambda_{1}^{*}+\lambda_{2}^{*}+\cdots+\lambda_{i}^{*} \leq \mu_{1}^{*}+\mu_{2}^{*}+\cdots+\mu_{i}^{*} \quad \forall i .
\end{aligned}
$$

The three relations (containment, domination and co-domination) define partial orders. We are mostly interested in the order of co-domination. Given a positive integer $r$, we denote by $\bar{\Lambda}_{r}$ (respectively $\Lambda_{r}$ ) the poset of pre-partitions (resp. partitions) of length at most $r$ with the order of co-domination. By $\Lambda_{r, n}$ we denote the poset of partitions of length at most $r$ and co-length at most $n$. It can be shown that $\bar{\Lambda}_{r}, \Lambda_{r}$ and $\Lambda_{r, n}$ are all lattices.

2.4. Adjacencies. In Section 4 we will need to have a good understanding of the structure of the posets $\bar{\Lambda}_{r}$. For our purposes, it will be enough to determine the adjacencies in $\bar{\Lambda}_{r}$.

Let $\lambda$ and $\mu$ be two different pre-partitions in $\bar{\Lambda}_{r}$ such that $\lambda \triangleleft \mu$. We say that $\lambda$ and $\mu$ are adjacent (or that $\mu$ covers $\lambda$ ) if there is no pre-partition $\nu$ in $\bar{\Lambda}_{r}$, different from $\lambda$ and $\mu$, such that $\lambda \triangleleft \nu \triangleleft \mu$. Adjacencies in $\Lambda_{r}$ were determined in dCEP80, Prop. 1.2]. They come in three different types, which we call single removals, slips and falls.

- We say that a pre-partition $\lambda$ is obtained from $\mu$ via a single removal if $\lambda_{i}=\mu_{i}$ for all $i \neq j$ and, $\lambda_{j}=\mu_{j}-1$, where $j$ is the smallest integer such that $\mu_{j}$ is finite. At the level of diagrams, $\lambda$ is obtained from $\mu$ by removing one box in the lowest row of finite size. Notice that this removal can only be done if $\mu_{j+1}<\mu_{j}$.

- We say that $\lambda$ is obtained from $\mu$ via a slip if there exists a positive integer $j$ such that $\lambda_{j+1}=\mu_{j+1}-1, \lambda_{j}=\mu_{j}+1$ and $\lambda_{i}=\mu_{i}$ for all $i \notin\{j, j+1\}$. In this case, the diagram of $\lambda$ is obtained from the diagram of $\mu$ by moving a box from row $j+1$ to row $j$. A slip from row $j+1$ can only happen if $\mu_{j+2}<\mu_{j+1}$, and $\mu_{j}<\mu_{j-1}$.

- We say that $\lambda$ is obtained from $\mu$ via a fall if $\mu^{*}$ is obtained from $\lambda^{*}$ via a slip. In other words, there exist positive integers $j<k$ such that $\lambda_{k}=\mu_{k}-1, \lambda_{j}=\mu_{j}+1$, and $\lambda_{i}=\mu_{i}$ for all $i \notin\{j, k\}$. A fall from row $k$ to row $j$ can only happen if $\mu_{k+1}<\mu_{k}, \mu_{j}<\mu_{j-1}$, and $\mu_{i}=\mu_{i^{\prime}}$ for all $i, i^{\prime} \in\{j, j+1, \ldots, k\}$. During a fall, a box in the diagram of $\mu$ is moved from one column to the next.

Since we are dealing with pre-partitions, we will also need to consider infinite removals.

- We say that a pre-partition $\lambda$ is obtained from $\mu$ via an infinite removal if $\lambda_{i}=\mu_{i}$ for all $i \neq j$ and $\lambda_{j}<\mu_{j}=\infty$, where $j$ is the largest integer such that $\mu_{j}$ is infinite. At the level of diagrams, $\lambda$ is obtained from $\mu$ by removing infinitely many boxes in the highest row of infinite size.

In dCEP80 the authors show that adjacencies in the set of partitions with respect to the order of domination correspond to simple removals, slips and falls. The result for partitions with the order of co-domination follows immediately from the fact that $\lambda \triangleleft \mu \Leftrightarrow \lambda^{*} \leq \mu^{*}$. Now consider two pre-partitions $\lambda \triangleleft \mu$ with finite 


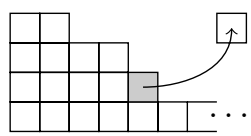

Single removal

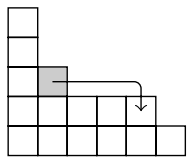

Slip

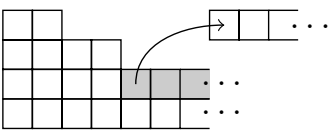

Infinite removal

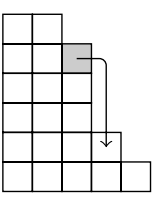

Fall

length, and assume they are adjacent. They must have the same number of infinite terms, otherwise the pre-partition $\nu$ obtained from $\lambda$ by adding one box in the lowest finite row verifies $\lambda \triangleleft \nu \triangleleft \mu$. Let $\hat{\lambda}$ and $\hat{\mu}$ be the partitions obtained from $\lambda$ and $\mu$ by removing the infinite terms. Then $\hat{\lambda}$ and $\hat{\mu}$ are adjacent and we can apply the result of [CEP80] to show that $\lambda$ can be obtained from $\mu$ via a simple removal, a slip, or a fall.

Theorem 2.1. Let $\lambda$ and $\mu$ be two pre-partitions in $\bar{\Lambda}_{r}$, and assume that $\lambda \triangleleft \mu$. Then there exists a finite sequence of pre-partitions in $\bar{\Lambda}_{r}$,

$$
\lambda=\nu^{m} \triangleleft \cdots \triangleleft \nu^{1} \triangleleft \nu^{0}=\mu,
$$

such that $\nu^{i}$ is obtained from $\nu^{i-1}$ via a simple removal, an infinite removal, a slip, or a fall.

Proof. Assume first that there are more infinite terms in $\mu$ that in $\lambda$. To each infinite row $j$ in $\mu$ which is finite in $\lambda$ we apply an infinite removal, leaving at least $\lambda_{j}$ boxes (depending on the particular $\mu$ we might need to leave more boxes). This way we obtain a sequence

$$
\lambda \triangleleft \nu^{m_{0}} \triangleleft \cdots \triangleleft \nu^{1} \triangleleft \nu^{0}=\mu,
$$

where $\lambda$ has the same number of infinite rows as $\nu^{m_{0}}$ and where each $\nu^{i}$ is obtained from $\nu^{i-1}$ via an infinite removal. Notice that $m_{0}$ is the number of rows which are infinite in $\mu$ but finite in $\lambda$. Since $\mu$ has finite length, $m_{0}$ is finite.

Let $k$ be the number of boxes in the finite rows of $\nu^{m_{0}}$. Then any pre-partition with the same number of infinite rows as $\nu^{m_{0}}$ and co-dominated by $\nu^{m_{0}}$ must use at most $k$ boxes in its finite rows. In particular, there are only finitely many such pre-partitions. It follows that we can find a finite sequence

$$
\lambda=\nu^{m} \triangleleft \cdots \triangleleft \nu^{m_{0}+1} \triangleleft \nu^{m_{0}}
$$

where consecutive terms are adjacent. From the discussion preceding the theorem, we see that $\nu^{i}$ can be obtained from $\nu^{i-1}$ by a simple removal, a slip, or a fall, and the result follows. 


\section{Orbit decomposition of the ARC SPACE}

We start by recalling our basic setup from the introduction. $\mathcal{M}=\mathbf{A}^{\text {rs }}$ is the space of $r \times s$ matrices with coefficients in $\mathbf{C}$, and we assume that $r \leq s$. The ring of regular functions on $\mathcal{M}$ is a polynomial ring on the entries of a generic matrix $x$ :

$$
\mathcal{O}_{\mathcal{M}}=\mathbf{C}\left[x_{11}, \ldots, x_{r s}\right], \quad x=\left(\begin{array}{cccc}
x_{11} & x_{12} & \ldots & x_{1 s} \\
x_{21} & x_{22} & \ldots & x_{2 s} \\
\vdots & \vdots & \ddots & \vdots \\
x_{r 1} & x_{r 2} & \ldots & x_{r s}
\end{array}\right) .
$$

The generic determinantal variety of matrices of rank at most $k$ is denoted by $D^{k}$. The ideal of $D^{k}$ is generated by the $(k+1) \times(k+1)$ minors of $x$. It can be shown that generic determinantal varieties are irreducible, and that the singular locus of $D^{k}$ is $D^{k-1}$ when $0<k<r$ ( $D^{0}$ and $D^{r}$ are smooth). They are also Cohen-Macaulay, Gorenstein, and have rational singularities. For proofs of the previous statements, and a comprehensive account of the theory of determinantal varieties, we refer the reader to BV88.

We denote by $G$ the group $\mathrm{GL}_{r} \times \mathrm{GL}_{s}$. It acts naturally on $\mathcal{M}$ via a change of basis:

$$
(g, h) \cdot A=g A h^{-1}, \quad(g, h) \in G, \quad A \in \mathcal{M} .
$$

The rank of a matrix is the only invariant for this action, and the determinantal varieties are the orbit closures (their ideals being the only invariant prime ideals of $\left.\mathcal{O}_{\mathcal{M}}\right)$.

The group $G$ is an algebraic group. In particular, it is an algebraic variety, and we can consider its arc space $G_{\infty}$ and its jet schemes $G_{n}$. The action of $G$ on $\mathcal{M}$ and $D^{k}$ induces actions at the level of arc spaces and jet schemes:

$$
\begin{array}{ll}
G_{\infty} \times \mathcal{M}_{\infty} \rightarrow \mathcal{M}_{\infty}, & G_{\infty} \times D_{\infty}^{k} \rightarrow D_{\infty}^{k}, \\
G_{n} \times \mathcal{M}_{n} \rightarrow \mathcal{M}_{n}, & G_{n} \times D_{n}^{k} \rightarrow D_{n}^{k} .
\end{array}
$$

In this section we classify the orbits associated to all of these actions.

As a set, the arc space $\mathcal{M}_{\infty}$ contains matrices of size $r \times s$ with entries in the power series ring $\mathbf{C} \llbracket t \rrbracket$. Analogously, the group $G_{\infty}=\left(\mathrm{GL}_{r}\right)_{\infty} \times\left(\mathrm{GL}_{s}\right)_{\infty}$ is formed by pairs of square matrices with entries in $\mathbf{C} \llbracket t \rrbracket$ which are invertible, that is, their determinant is a unit in $\mathbf{C} \llbracket t \rrbracket$. Orbits in $\mathcal{M}_{\infty}$ correspond to similarity classes of matrices over the ring $\mathbf{C} \llbracket t \rrbracket$, and we can easily classify these using the fact that $\mathbf{C} \llbracket t \rrbracket$ is a principal ideal domain.

Definition 3.1 (Orbit associated to a partition). Let $\lambda=\left(\lambda_{1}, \lambda_{2}, \ldots, \lambda_{r}\right) \in \bar{\Lambda}_{r}$ be a pre-partition with length at most $r$. Consider the following matrix in $M_{\infty}$ :

$$
\delta_{\lambda}=\left(\begin{array}{ccccccc}
0 & \cdots & 0 & t^{\lambda_{1}} & 0 & \cdots & 0 \\
0 & \cdots & 0 & 0 & t^{\lambda_{2}} & \cdots & 0 \\
\vdots & & \vdots & \vdots & \vdots & \ddots & \vdots \\
0 & \cdots & 0 & 0 & 0 & \cdots & t^{\lambda_{r}}
\end{array}\right)
$$

(we use the convention that $t^{\infty}=0$ ). Then the $G_{\infty}$-orbit of the matrix $\delta_{\lambda}$ is called the orbit in $\mathcal{M}_{\infty}$ associated to the pre-partition $\lambda$, and it is denoted by $\mathcal{C}_{\lambda}$.

Proposition 3.2 (Orbits in $\mathcal{M}_{\infty}$ ). Every $G_{\infty}$-orbit of $\mathcal{M}_{\infty}$ is of the form $\mathcal{C}_{\lambda}$ for some pre-partition $\lambda \in \bar{\Lambda}_{r}$. An orbit $\mathcal{C}_{\lambda}$ is contained in $D_{\infty}^{k}$ if and only if the 
associated pre-partition $\lambda$ contains at least $r-k$ leading infinities, i.e. $\lambda_{1}=\cdots=$ $\lambda_{r-k}=\infty$. In particular, $\mathcal{M}_{\infty} \backslash D_{\infty}^{r-1}$ is the union of the orbits corresponding to partitions, and the orbits in $D_{\infty}^{k} \backslash D_{\infty}^{k-1}$ are in bijection with $\Lambda_{k}$. Moreover, the orbit corresponding to the empty partition $(0,0, \ldots)$ is the arc space $\left(\mathcal{M} \backslash D^{r-1}\right)_{\infty}$.

Proof. As mentioned above, $M_{\infty}$ is the set of $r \times s$-matrices with coefficients in the ring $\mathbf{C} \llbracket t \rrbracket$, and the group $G_{\infty}$ acts on $M_{\infty}$ via row and column operations, also with coefficients in $\mathbf{C} \llbracket t \rrbracket$. Using Gaussian elimination and the fact that $\mathbf{C} \llbracket t \rrbracket$ is a principal ideal domain, we see that each $G_{\infty}$-orbit in $M_{\infty}$ contains a matrix of the form

$$
\left(\begin{array}{ccccccc}
0 & \cdots & 0 & t^{\lambda_{1}} & 0 & \cdots & 0 \\
0 & \cdots & 0 & 0 & t^{\lambda_{2}} & \cdots & 0 \\
\vdots & & \vdots & \vdots & \vdots & \ddots & \vdots \\
0 & \cdots & 0 & 0 & 0 & \cdots & t^{\lambda_{r}}
\end{array}\right)
$$

where the exponents $\lambda_{i}$ are non-negative integers or $\infty$, and we use the convention that $t^{\infty}=0$. After permuting columns and rows, we can assume that the exponents of these powers form a non-increasing sequence:

$$
\lambda_{1} \geq \lambda_{2} \geq \cdots \geq \lambda_{r} .
$$

Moreover, the usual structure theorems for finitely generated modules over principal ideal domains guarantee that each orbit contains a unique matrix in this form. This shows that the set of $G_{\infty}$-orbits in $M_{\infty}$ is in bijection with $\bar{\Lambda}_{r}$.

The ideal defining $D^{k}$ in $\mathcal{M}$ is generated by the minors of size $(k+1) \times(k+1)$. Let $\lambda \in \bar{\Lambda}_{r}$ be a pre-partition of length at most $r$ and consider $\delta_{\lambda}$ as in Definition 3.1. The $(k+1) \times(k+1)$ minors of $\delta_{\lambda}$ are either zero or of the form $\prod_{i \in I} t^{\lambda_{i}}$, where $I$ is a subset of $\{1, \ldots, r\}$ with $k+1$ elements. For all of the minors to be zero, we need at least $r-k$ infinities in the set $\left\{\lambda_{1}, \ldots, \lambda_{r}\right\}$ (recall that $t^{\infty}=0$ ). In other words, $\delta_{\lambda}$ is contained in $D_{\infty}^{k}$ if and only if $\lambda$ contains $r-k$ leading infinities.

The variety $D^{k}$ is invariant under the action of $G$, so $D_{\infty}^{k}$ is invariant under the action of $G_{\infty}$. In particular, the orbit $\mathcal{C}_{\lambda}$ is contained in $D_{\infty}^{k}$ if and only if $\delta_{\lambda}$ is contained in $D_{\infty}^{k}$. The rest of the proposition follows immediately.

Proposition 3.3 (Orbits and contact loci). The contact locus $\operatorname{Cont}^{p}\left(D^{k}\right)$ is invariant under the action of $G_{\infty}$, and the orbits contained in $\operatorname{Cont}^{p}\left(D^{k}\right)$ correspond to those pre-partitions $\lambda \in \bar{\Lambda}_{r}$ whose last $k+1$ terms add up to at least $p$ :

$$
\lambda_{r-k}+\cdots+\lambda_{r} \geq p .
$$

Proof. The truncation maps from the arc space to the jet schemes are in fact natural transformations of functors. This means that we have the following natural diagram:

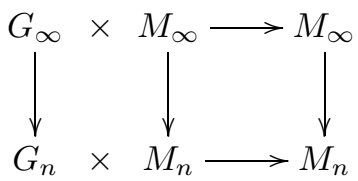

Since $D^{k}$ is $G$-invariant, $D_{n}^{k}$ is $G_{n}$-invariant, so $\operatorname{Cont}^{p}\left(D^{k}\right)$ (the inverse image of $D_{p-1}^{k}$ under the truncation map) is $G_{\infty}$-invariant. In particular, an orbit $\mathcal{C}_{\lambda}$ is 
contained in $\operatorname{Cont}^{p}\left(D^{k}\right)$ if and only if its base point $\delta_{\lambda}$ is contained in $\operatorname{Cont}^{p}\left(D^{k}\right)$. The order of vanishing of $\mathcal{I}_{D^{k}}$ along $\delta_{\lambda}$ is $\lambda_{r-k}+\cdots+\lambda_{r}$ (recall that $\mathcal{I}_{D^{k}}$ is generated by the minors of size $(k+1) \times(k+1)$ and that $\left.\lambda_{1} \geq \cdots \geq \lambda_{r}\right)$. Hence $\delta_{\lambda}$ belongs to $\operatorname{Cont}^{p}\left(D^{k}\right)$ if and only if $\lambda_{r-k}+\cdots+\lambda_{r} \geq p$, and the proposition follows.

Proposition 3.4 (Orbits are cylinders). Let $\lambda \in \bar{\Lambda}_{r}$ be a pre-partition, and let $\mathcal{C}_{\lambda}$ be the associated orbit in $\mathcal{M}_{\infty}$. If $\lambda$ is a partition, $\mathcal{C}_{\lambda}$ is a cylinder of $\mathcal{M}_{\infty}$. More generally, let $r-k$ be the number of infinite terms of $\lambda$. Then $\mathcal{C}_{\lambda}$ is a cylinder of $D_{\infty}^{k}$

Proof. Assume that $\lambda$ is a pre-partition with $r-k$ leading infinities, and consider the following cylinders in $\mathcal{M}_{\infty}$ :

$$
\begin{aligned}
& A_{\lambda}=\operatorname{Cont}^{\lambda_{r}}\left(D^{0}\right) \cap \operatorname{Cont}^{\lambda_{r}+\lambda_{r-1}}\left(D^{1}\right) \cap \operatorname{Cont}^{\lambda_{r}+\cdots+\lambda_{r-k}}\left(D^{k}\right), \\
& B_{\lambda}=\operatorname{Cont}^{\lambda_{r}+1}\left(D^{0}\right) \cup \operatorname{Cont}^{\lambda_{r}+\lambda_{r-1}+1}\left(D^{1}\right) \cup \operatorname{Cont}^{\lambda_{r}+\cdots+\lambda_{r-k}+1}\left(D^{k}\right) .
\end{aligned}
$$

By Propositions 3.2 and 3.3 , we know that

$$
\mathcal{C}_{\lambda}=\left(A_{\lambda} \backslash B_{\lambda}\right) \cap D_{\infty}^{k} .
$$

Hence $\mathcal{C}_{\lambda}$ is a cylinder in $D_{\infty}^{k}$, as required.

Remark 3.5. The proof of Proposition 3.4 tells us that we can express all orbits in $\mathcal{M}_{\infty}$ with contact conditions with respect to the determinantal varieties. In particular, if we know the order of contact of an arc with respect to all determinantal varieties, we know which orbit it belongs to. Moreover, this is also true not only for closed points, but for all schematic points of $\mathcal{M}_{\infty}$. It follows that every point of $\mathcal{M}_{\infty}$ (closed or not) is contained in an orbit generated by a closed point.

We now study the jet schemes $G_{n}$ and $\mathcal{M}_{n}$. As in the case of the arc space, elements in $G_{n}$ and $\mathcal{M}_{n}$ are given by matrices, but now the coefficients lie in the $\operatorname{ring} \mathbf{C}[t] /\left(t^{n+1}\right)$.

Definition 3.6. Let $\lambda=\left(\lambda_{1}, \ldots, \lambda_{\ell}\right) \in \Lambda_{r, n+1}$ be a partition with length at most $r$ and co-length at most $n+1$. Then the matrix $\delta_{\lambda}$ considered in Definition 3.1 gives an element of the jet scheme $\mathcal{M}_{n}$. The $G_{n}$-orbit of $\delta_{\lambda}$ is called the orbit of $\mathcal{M}_{n}$ associated to $\lambda$ and is denoted by $\mathcal{C}_{\lambda, n}$.

Proposition 3.7 (Orbits in $\mathcal{M}_{n}$ ). Every $G_{n}$-orbit of $\mathcal{M}_{n}$ is of the form $\mathcal{C}_{\lambda, n}$ for some partition $\lambda \in \Lambda_{r, n+1}$. An orbit $\mathcal{C}_{\lambda, n}$ is contained in $D_{n}^{k}$ if and only if the associated partition contains at least $r-k$ terms equal to $n+1$. In particular, the set of orbits in $D_{n}^{k} \backslash D_{n}^{k-1}$ is in bijection with $\Lambda_{k, n}$.

Proof. This can be proven in the same way as Proposition 3.2. The only difference is that we now work with a principal ideal ring $\mathbf{C}[t] /\left(t^{n+1}\right)$, as opposed to the principal ideal domain $\mathbf{C} \llbracket t \rrbracket$, but the domain condition played no role in the proof of Proposition 3.2. Alternatively, one can notice that $\mathbf{C}[t] /\left(t^{n+1}\right)$ is a quotient of $\mathbf{C} \llbracket t \rrbracket$, so modules over $\mathbf{C}[t] /\left(t^{n+1}\right)$ correspond to modules over $\mathbf{C} \llbracket t \rrbracket$ with the appropriate annihilator, and one can reduce the problem of classifying $G_{n}$-orbits in $\mathcal{M}_{n}$ to classifying $G_{\infty}$-orbits in $\mathcal{M}_{\infty}$ with bounded exponents. 
Definition 3.8. Let $\lambda=\left(\lambda_{1}, \lambda_{2}, \ldots\right)$ be a pre-partition, and let $n$ be a non-negative integer. Then the truncation of $\lambda$ to level $n$ is the partition $\bar{\lambda}=\left(\bar{\lambda}_{1}, \bar{\lambda}_{2}, \ldots\right)$ where

$$
\bar{\lambda}_{i}=\min \left(\lambda_{i}, n\right) \text {. }
$$

Proposition 3.9 (Truncation of orbits). Let $\lambda \in \bar{\Lambda}_{r}$ be a pre-partition, and let $\bar{\lambda}$ be its truncation to level $n+1$. Then the image of $\mathcal{C}_{\lambda}$ under the natural truncation map $\mathcal{M}_{\infty} \rightarrow \mathcal{M}_{n}$ is $\mathcal{C}_{\bar{\lambda}, n}$. Conversely, fix a partition $\bar{\lambda} \in \Lambda_{r, n+1}$, and let $\Gamma \subset \bar{\Lambda}_{r}$ be the set of pre-partitions whose truncation to level $n+1$ is $\bar{\lambda}$. Then the inverse image of $\mathcal{C}_{\bar{\lambda}, n}$ under the truncation map is the union of the orbits of $\mathcal{M}_{\infty}$ corresponding to the pre-partitions in $\Gamma$.

Proof. Notice that $\delta_{\bar{\lambda}} \in \mathcal{M}_{n}$ is the truncation of $\delta_{\lambda} \in \mathcal{M}_{\infty}$. Then the fact that the truncation of $\mathcal{C}_{\lambda}=G_{\infty} \cdot \delta_{\lambda}$ equals $\mathcal{C}_{\bar{\lambda}, n}=G_{n} \cdot \delta_{\bar{\lambda}}$ is an immediate consequence of the fact that the truncation map is a natural transformation of functors (see the proof of Proposition 3.3). Conversely, if $\lambda$ and $\lambda^{\prime}$ have different truncations, the $G_{n}$-orbits $\mathcal{C}_{\bar{\lambda}, n}$ and $\mathcal{C}_{\bar{\lambda}^{\prime}, n}$ are different, so $\mathcal{C}_{\lambda^{\prime}}$ is not in the fiber of $\mathcal{C}_{\bar{\lambda}, n}$.

\section{ORBIT POSET AND IRREDUCIBLE COMPONENTS OF JET SCHEMES}

After obtaining a classification of the orbits of the action of $G_{\infty}=\left(\mathrm{GL}_{r}\right)_{\infty} \times$ $\left(\mathrm{GL}_{s}\right)_{\infty}$ on $\mathcal{M}_{\infty}$ and $D_{\infty}^{k}$, we start the study of their geometry. The first basic question is the following: how are these orbits positioned with respect to each other inside the arc space $\mathcal{M}_{\infty}$ ? We can make this precise by introducing the notion of an orbit poset.

Definition 4.1 (Orbit poset). Let $\mathcal{C}$ and $\mathcal{C}^{\prime}$ be two $G_{\infty}$-orbits in $\mathcal{M}_{\infty}$. We say that $\mathcal{C}$ dominates $\mathcal{C}^{\prime}$, and denote it by $\mathcal{C}^{\prime} \leq \mathcal{C}$, if $\mathcal{C}^{\prime}$ is contained in the Zariski closure of $\mathcal{C}$. The relation of dominance defines a partial order on the set of $G_{\infty}$-orbits of $\mathcal{M}_{\infty}$. The pair $\left(\mathcal{M}_{\infty} / G_{\infty}, \leq\right)$ is known as the orbit poset of $\mathcal{M}_{\infty}$.

Our goal is to prove that the bijection that maps a pre-partition to its associated orbit in $\mathcal{M}_{\infty}$ is in fact an order-reversing isomorphism between $\Lambda_{r}$ and the orbit poset. At this stage it is not hard to show that one of the directions of this bijection reverses the order.

Proposition 4.2 (Domination of orbits implies co-domination of partitions). Let $\lambda, \lambda^{\prime} \in \bar{\Lambda}_{r}$ be two pre-partitions of length at most $r$, and let $\mathcal{C}_{\lambda}$ and $\mathcal{C}_{\lambda^{\prime}}$ be the associated orbits in $\mathcal{M}_{\infty}$. If $\mathcal{C}_{\lambda}$ dominates $\mathcal{C}_{\lambda^{\prime}}$, then $\lambda^{\prime}$ co-dominates $\lambda$ :

$$
\mathcal{C}_{\lambda} \geq \mathcal{C}_{\lambda^{\prime}} \quad \Longrightarrow \quad \lambda \triangleleft \lambda^{\prime} \text {. }
$$

Proof. From Proposition 3.3 we get that $\mathcal{C}_{\lambda} \subset \operatorname{Cont}^{p}\left(D^{k}\right)$, where $p=\lambda_{r}+\cdots+\lambda_{r-k}$. Since a contact locus is always Zariski closed, if $\mathcal{C}_{\lambda}$ dominates $\mathcal{C}_{\lambda^{\prime}}$ we also know that $\mathcal{C}_{\lambda^{\prime}} \subset \operatorname{Cont}^{p}\left(D^{k}\right)$. Again by Proposition 3.3, this gives $\lambda_{r}^{\prime}+\cdots+\lambda_{r-k}^{\prime} \geq p$, as required.

We now proceed to prove the converse to Proposition 4.2 Given two prepartitions $\lambda, \lambda^{\prime} \in \bar{\Lambda}_{r}$ with $\lambda \triangleleft \lambda^{\prime}$, we need to show that the closure of $\mathcal{C}_{\lambda}$ contains $\mathcal{C}_{\lambda^{\prime}}$. We will exhibit this containment by producing a "path" in the $\operatorname{arc}$ space $\mathcal{M}_{\infty}$ whose general point is in $\mathcal{C}_{\lambda}$ but specializes to a point in $\mathcal{C}_{\lambda^{\prime}}$. These types of "paths" are known as wedges. 
Definition 4.3 (Wedge). Let $X$ be a scheme over C. A wedge $w$ on $X$ is a morphism of schemes $w: \operatorname{Spec} \mathbf{C} \llbracket s, t \rrbracket \rightarrow X$. Given a wedge $w$, one can consider the diagram

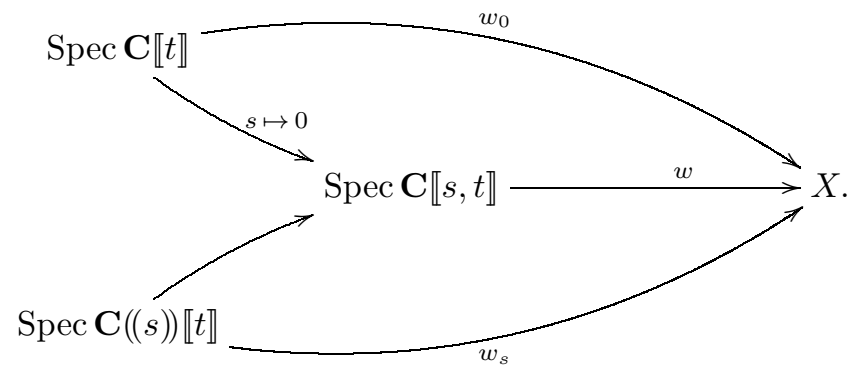

The map $w_{0}$ is known as the special arc of $w$, and $w_{s}$ as the generic arc of $w$.

Lemma 4.4. Let $w$ be a wedge on $\mathcal{M}$ and let $\mathcal{C}_{0}$ be the $G_{\infty}$-orbit in $\mathcal{M}_{\infty}$ of the special arc $w_{0}$ of $w$. Assume that there is a $G_{\infty}$-orbit $\mathcal{C}_{s}$ in $\mathcal{M}_{\infty}$ containing the generic arc $w_{s}$ of $w$. Then $\mathcal{C}_{s}$ dominates $\mathcal{C}_{0}$.

Proof. By the hypothesis, the closure of $\mathcal{C}_{s}$ contains $w_{0}$ (because $w_{0}$ is in the closure of $w_{s}$ ). But the closure of an orbit is invariant, so $\overline{\mathcal{C}_{s}}$ must contain $\mathcal{C}_{0}=G_{\infty} \cdot w_{0}$.

Lemma 4.5. Let $\lambda$ and $\mu$ be two pre-partitions in $\bar{\Lambda}_{r}$ and assume that $\lambda$ is obtained from $\mu$ via a removal (simple or infinite). Then $\mathcal{C}_{\lambda}$ dominates $\mathcal{C}_{\mu}$.

Proof. Let $i$ be the index such that $\lambda_{i}<\mu_{i}$, and consider the following wedge on $\mathcal{M}$ :

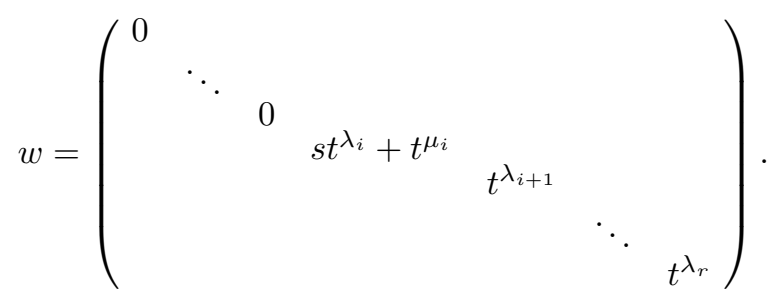

The special arc of $w$ is $\delta_{\mu}$. The generic arc $w_{s}$ only differs from $\delta_{\lambda}$ by the presence of the unit $s+t^{\mu_{i}-\lambda_{i}}$ on row $i$. Therefore $w_{s}$ and $\delta_{\lambda}$ have the same contact with respect to all the determinantal varieties, and the proof of Proposition 3.4 shows that $w_{s}$ is contained in $\mathcal{C}_{\lambda}$. Now we can apply Lemma 4 .4 with $\mathcal{C}_{0}=\mathcal{C}_{\mu}$ and $\mathcal{C}_{s}=\mathcal{C}_{\lambda}$, and the result follows.

Lemma 4.6. Let $\lambda$ and $\mu$ be two pre-partitions in $\bar{\Lambda}_{r}$ and assume that $\lambda$ is obtained from $\mu$ via a slip or a fall. Then $\mathcal{C}_{\lambda}$ dominates $\mathcal{C}_{\mu}$. 
Proof. Let $i<j$ be the indices such that $\lambda_{i}=\mu_{i}+1, \lambda_{j}=\mu_{j}-1$ and $\mu_{k}=\lambda_{k}$ for $k \neq i, j$. Consider the following wedge:

$$
w=\left(\begin{array}{ccccccccccc}
t^{\lambda_{1}} & & & & & & & & & & \\
& \ddots & & & & & & & & & \\
& & t^{\lambda_{i-1}} & & & & & & & & \\
& & & \alpha & 0 & \cdots & 0 & \beta & & & \\
& & & \vdots & \vdots & \ddots & \vdots & \vdots & & & \\
& & & 0 & 0 & \cdots & t^{\lambda_{j+1}+1} & 0 & & & \\
& & & \gamma & 0 & \cdots & 0 & \delta & & & \\
& & & & & & & & t^{\lambda_{j+1}} & & \\
& & & & & & & & & \ddots & \\
& & & & & & & & & & t^{\lambda_{r}}
\end{array}\right),
$$

where

Notice that

$$
\left(\begin{array}{ll}
\alpha & \beta \\
\gamma & \delta
\end{array}\right)=\left(\begin{array}{cc}
s t^{\lambda_{i}}+t^{\lambda_{i}-1} & t^{\lambda_{i}-1} \\
s t^{\lambda_{j}} & s t^{\lambda_{j}}+t^{\lambda_{j}+1}
\end{array}\right)
$$

$$
\begin{gathered}
\operatorname{ord}_{t}\left(\begin{array}{ll}
\alpha & \beta \\
\gamma & \delta
\end{array}\right)=\lambda_{j},\left.\quad \operatorname{ord}_{t}\left(\begin{array}{ll}
\alpha & \beta \\
\gamma & \delta
\end{array}\right)\right|_{s=0}=\lambda_{j}+1, \\
\operatorname{det}\left(\begin{array}{ll}
\alpha & \beta \\
\gamma & \delta
\end{array}\right)=t^{\lambda_{i}+\lambda_{j}}\left(1+s t+s^{2}\right) .
\end{gathered}
$$

From these equations, we see that $w_{0}$ and $\delta_{\mu}$ have the same order of contact with respect to all determinantal varieties, and the proof of Proposition 3.4 tells us that $w_{0}$ is contained in $\mathcal{C}_{\mu}$. Analogously, the equations above show that $w_{s}$ is contained in $\mathcal{C}_{\lambda}$. Lemma 4.4 gives the result.

Theorem 4.7 (Orbit poset $=$ Pre-partition poset). The map that sends a prepartition $\lambda \in \bar{\Lambda}_{r}$ of length at most $r$ to the associated orbit $\mathcal{C}_{\lambda}$ in $\mathcal{M}_{\infty}$ is an orderreversing isomorphism between $\bar{\Lambda}_{r}$ and the orbit poset:

$$
\mathcal{C}_{\lambda} \geq \mathcal{C}_{\mu} \quad \Longleftrightarrow \lambda \triangleleft \mu \quad \Longleftrightarrow \quad \lambda_{r-i}+\cdots+\lambda_{r} \leq \mu_{r-i}+\cdots+\mu_{r} \quad \forall i .
$$

Proof. The theorem follows from Proposition 4.2 Lemma 4.5, Lemma 4.6, and Theorem 2.1.

In the remainder of the section we use Theorem 4.7 to compute the number of irreducible components of the jet schemes of generic determinantal varieties.

Notation 4.8. As is customary in the theory of partitions, we write $\lambda=\left(d_{1}^{a_{1}} \ldots d_{j}^{a_{j}}\right)$ to denote the pre-partition that has $a_{i}$ copies of $d_{i}$. For example,

$$
(\infty, \infty, 5,3,3,3,2,1,1)=\left(\infty^{2} 5^{1} 3^{3} 2^{1} 1^{2}\right) .
$$

Proposition 4.9. Recall that $D^{k} \subset \mathcal{M}$ denotes the determinantal variety of matrices of size $r \times s$ and rank at most $k$. Assume that $0<k<r-1$, and let $\mathcal{C}$ be an irreducible component of $\operatorname{Cont}^{p}\left(D^{k}\right) \subset \mathcal{M}_{\infty}$. Then $\mathcal{C}$ contains a unique dense $G_{\infty}$-orbit $\mathcal{C}_{\lambda}$. Moreover, $\lambda$ is a partition (contains no infinite terms) and $\lambda=\left(d^{a+r-k} e^{1}\right)$ where

$$
p=(a+1) d+e, \quad 0 \leq e<d,
$$


and either $e=0$ and $0 \leq a \leq k$ or $e>0$ and $0 \leq a<k$. Conversely, for any partition as above, its associated orbit is dense in an irreducible component of $\operatorname{Cont}^{p}\left(D^{k}\right)$.

Example 4.10. When $r=8, k=6$, and $p=5$, the partitions given by the proposition are

$$
(5,5), \quad(4,4,1), \quad(3,3,2), \quad(2,2,2,1), \quad(1,1,1,1,1,1) .
$$

When $r=5, k=3$, and $p=5$, we only get

$$
(5,5), \quad(4,4,1), \quad(3,3,2), \quad(2,2,2,1) .
$$

Proof. By Theorem 4.7 and Proposition 3.3. computing the irreducible components of $\operatorname{Cont}^{p}\left(D^{k}\right)$ is equivalent to computing the minimal elements (with respect to the order of co-domination) among all pre-partitions $\lambda \in \bar{\Lambda}_{r}$ such that

$$
\lambda_{r}+\lambda_{r-1}+\cdots+\lambda_{r-k} \geq p .
$$

Let $\Sigma$ be the set of such partitions. To find minimal elements in $\Sigma$ it will be useful to keep in mind the structure of the adjacencies in $\bar{\Lambda}_{r}$ discussed in Section 2.4.

First notice that all minimal elements in $\Sigma$ must be partitions. Indeed, given an element $\lambda \in \Sigma$, truncating all infinite terms of $\lambda$ to a high enough number produces another element of $\Sigma$. Moreover, if $\lambda \in \Sigma$ is minimal, we must have $\lambda_{1}=\lambda_{2}=\cdots=\lambda_{r-k}$. If this were not the case, we could consider the partition $\lambda^{\prime}$ such that $\lambda_{1}^{\prime}=\cdots=\lambda_{r-k}^{\prime}=\lambda_{r-k}$, and $\lambda_{i}^{\prime}=\lambda_{i}$ for $i>r-k$. Then $\lambda^{\prime}$ would also be in $\Sigma$, but $\lambda^{\prime} \triangleleft \lambda$, contradicting the fact that $\lambda$ is minimal. It is also clear that minimal elements of $\Sigma$ must verify $\lambda_{r-k}+\cdots+\lambda_{r}=p$. In fact, if a partition in $\Sigma$ does not verify this, we can decrease the last terms of the partition and still remain in $\Sigma$.

So far we know that the minimal elements in $\Sigma$ are partitions that verify $\lambda_{1}=$ $\cdots=\lambda_{r-k}$ and $\lambda_{r-k}+\cdots+\lambda_{r}=p$. Note that we assume $0<k<r-1$, so for any two partitions $\lambda, \lambda^{\prime}$ with the previous properties, if $\lambda_{r-k} \neq \lambda_{r-k}^{\prime}$, then $\lambda$ and $\lambda^{\prime}$ are not comparable.

Pick a minimal element $\lambda \in \Sigma$, and write $d=\lambda_{r-k}$. Let $\ell$ be the length of $\lambda$. The proposition will follow if we show that the sequence $\left(\lambda_{r-k}, \lambda_{r-k+1}, \ldots, \lambda_{\ell}\right)$ is of the form $(d, \ldots, d, e)$ for some $0 \leq e<d$. But this is clear from the analysis of the adjacencies in $\bar{\Lambda}_{r}$ given in Section 2.4. Consider the Young diagram $\Gamma$ associated to $\lambda$. The longest row of $\Gamma$ has length $d$. If there are two rows, say $i<j$, with length less than $d$, then we must have $r-k<i$ and we can move one box from row $j$ to row $i$ (via a sequence of falls and slips) and obtain a partition still in $\Sigma$ but co-dominated by $\lambda$. This contradicts the fact that $\lambda$ is minimal, and we see that $\lambda$ must have the form given in the proposition.

Proposition 4.11. Assume that $k=0$ or $k=r-1$. Then $\operatorname{Cont}^{p}\left(D^{k}\right) \subset \mathcal{M}_{\infty}$ is irreducible and contains a unique dense orbit $\mathcal{C}_{\lambda}$, where $\lambda=\left(p^{r-k}\right)$.

Proof. From Proposition 3.3, the orbits $\mathcal{C}_{\lambda}$ contained in $\operatorname{Cont}^{p}\left(D^{0}\right)$ are the ones that verify $\lambda_{r} \geq p$. It is clear that the minimal partition of this type is $\left(p^{r}\right)$. Analogously, $\operatorname{Cont}^{p}\left(D^{r-1}\right)$ contains orbits whose associated partitions verify $\lambda_{1}+\cdots+\lambda_{r} \geq p$, and the minimal one among these is $\left(p^{1}\right)$. 
Theorem 4.12. If $k=0$ or $k=r-1$, the contact locus $\operatorname{Cont}^{p}\left(D^{k}\right) \subset \mathcal{M}_{\infty}$ is irreducible. Otherwise, the number of irreducible components of $\operatorname{Cont}^{p}\left(D^{k}\right) \subset \mathcal{M}_{\infty}$ is

$$
p+1-\left\lceil\frac{p}{k+1}\right\rceil .
$$

Proof. The first assertion follows directly from Proposition 4.11, For the second one, we need to count the number of partitions that appear in Proposition 4.9. Recall that these were partitions of the form $\lambda^{d}=\left(d^{a+r-k}, e^{1}\right)$ of length at most $r$ such that $p=(a+1) d+e$ and $0 \leq e<d$. Since $d$ ranges from 0 to $p$, we have at most $p+1$ such partitions. But as we decrease $d$, the length of $\lambda^{d}$ increases, possibly surpassing the limit $r$. Therefore the number of allowed partitions is $p+1-d_{0}$, where $d_{0}$ is the smallest integer such that $\lambda^{d_{0}}$ has length no greater than $r$.

If $d$ divides $p$, the length of $\lambda^{d}$ is $\left(\frac{p}{d}-1+r-k\right)$. Otherwise it is $\left(\left\lfloor\frac{p}{d}\right\rfloor+r-k\right)$. In either case, the length is no greater than $r$ if and only if $d \geq\left\lceil\frac{p}{k+1}\right\rceil$. Hence $d_{0}=\left\lceil\frac{p}{k+1}\right\rceil$, and the theorem follows.

Corollary 4.13. If $k=0$ or $k=r-1$, the jet scheme $D_{n}^{k}$ is irreducible. Otherwise, the number of irreducible components of $D_{n}^{k}$ is

$$
n+2-\left\lceil\frac{n+1}{k+1}\right\rceil .
$$

Proof. The contact locus Cont ${ }^{n+1}\left(D^{k}\right)$ is the inverse image of the jet scheme $D_{n}^{k}$ under the truncation map $\mathcal{M}_{\infty} \rightarrow \mathcal{M}_{n}$. Since $\mathcal{M}$ is smooth, this truncation map is surjective, so $D_{n}^{k}$ has the same number of components as $\operatorname{Cont}^{n+1}\left(D^{k}\right)$. Now the result follows directly from Theorem 4.12 ,

\section{Discrepancies and log Canonical thresholds}

In this section we compute discrepancies for all invariant divisorial valuations over $\mathcal{M}$ and over $D^{k}$, and we use it to give formulas for log canonical thresholds involving determinantal varieties. We start with a proposition that determines all possible invariant maximal divisorial sets in terms of orbits in the arc space.

Proposition 5.1 (Divisorial sets $=$ Orbit closures, $\mathcal{M}$ ). Let $\nu$ be a $G$-invariant divisorial valuation over $\mathcal{M}$, and let $\mathcal{C}$ be the associated maximal divisorial set in $\mathcal{M}_{\infty}$. Then there exists a unique partition $\lambda \in \Lambda_{r}$ of length at most $r$ whose associated orbit $\mathcal{C}_{\lambda}$ is dense in $\mathcal{C}$. Conversely, the closure of $\mathcal{C}_{\lambda}$, where $\lambda$ is a partition, is a maximal divisorial set associated to an invariant valuation.

Proof. Recall from Section 1.2 (or see [sh08]) that $\mathcal{C}$ is the union of the fat sets of $\mathcal{M}_{\infty}$ that induce the valuation $\nu$. Therefore, since $\nu$ is $G$-invariant, $\mathcal{C}$ is $G_{\infty^{-}}$ invariant and can be written as a union of orbits. Note that the thin orbits of $\mathcal{M}_{\infty}$ are all contained in $D_{\infty}^{r-1}$, and that $\mathcal{C}$ is itself fat, so $\mathcal{C}$ must contain a fat orbit. Let $\Sigma \subset \Lambda_{r}$ be the set of partitions indexing fat orbits contained in $\mathcal{C}$. For $\mu \in \Sigma$ we denote by $\nu_{\mu}$ the valuation induced by $\mathcal{C}_{\mu}$. Then, for $f \in \mathcal{O}_{\mathcal{M}}$ we have

$$
\nu(f)=\min _{\gamma \in \mathcal{C}}\left\{\operatorname{ord}_{\gamma}(f)\right\}=\min _{\mu \in \Sigma} \min _{\gamma \in \mathcal{C}_{\mu}}\left\{\operatorname{ord}_{\gamma}(f)\right\}=\min _{\mu \in \Sigma}\left\{\nu_{\mu}(f)\right\} .
$$


As a consequence, since $\nu_{\mu}$ is determined by its value on the ideals $\mathcal{I}_{D^{0}}, \ldots, \mathcal{I}_{D^{r-1}}$, the same property holds for $\nu$. Let $\lambda=\left(\lambda_{1}, \ldots, \lambda_{r}\right)$ be such that $\nu\left(\mathcal{I}_{D^{k}}\right)=\lambda_{r}+$ $\cdots+\lambda_{r-k}$. From the fact that $\mathcal{I}_{D^{k}} \mathcal{I}_{D^{k-2}} \subset \mathcal{I}_{D^{k-1}}^{2}$ we deduce that $\lambda_{k} \geq \lambda_{k+1}$, and we get a partition $\lambda \in \Lambda_{r}$ whose associated orbit $\mathcal{C}_{\lambda}$ induces the valuation $\nu$ (so $\lambda \in \Sigma$ ). The proposition follows if we show that $\mathcal{C}_{\lambda}$ is dense in $\mathcal{C}$.

Consider $\mu \in \Sigma$. Since $\mathcal{C}_{\mu} \subset \mathcal{C}$, we know that $\nu_{\mu} \geq \nu$, and we get that

$$
\mu_{r}+\cdots+\mu_{r-k}=\nu_{\mu}\left(\mathcal{I}_{D^{k}}\right) \geq \nu\left(\mathcal{I}_{D^{k}}\right)=\lambda_{r}+\cdots+\lambda_{r-k} .
$$

Hence $\lambda \triangleleft \mu$, and Theorem 4.7 tells us that $\mathcal{C}_{\mu}$ is contained in the closure of $\mathcal{C}_{\lambda}$, as required.

Proposition 5.2 (Divisorial sets $=$ Orbit closures, $\left.D^{k}\right)$. Given a partition $\lambda=$ $\left(\lambda_{1}, \ldots, \lambda_{\ell}\right) \in \Lambda_{k}$ of length at most $k$, denote by $\lambda^{+}=\left(\infty, \ldots, \infty, \lambda_{1}, \ldots, \lambda_{\ell}\right) \in \bar{\Lambda}_{r}$ the pre-partition obtained by adjoining $r-k$ infinities. Let $\nu$ be a $G$-invariant divisorial valuation over $D^{k}$, and let $\mathcal{C}$ be the associated maximal divisorial set in $D_{\infty}^{k}$. Then there exists a unique partition $\lambda \in \Lambda_{k}$ such that the orbit $\mathcal{C}_{\lambda^{+}}$is dense in $\mathcal{C}$. Conversely, the closure of $\mathcal{C}_{\lambda^{+}}$, where $\lambda \in \Lambda_{k}$, is a maximal divisorial set in $D_{\infty}^{k}$ associated to a $G$-invariant divisorial valuation.

Proof. Analogous to the proof of Proposition 5.1.

We now proceed to compute discrepancies for invariant divisorial valuations. These are closely related to the codimensions of the corresponding maximal divisorial sets, which by the previous propositions are just given by orbit closures. Since orbits are cylinders, their codimension can be computed by looking at the corresponding orbit in a high enough jet scheme. But jet schemes are of finite type, so orbits have a finite dimension that can be computed via the codimension of the corresponding stabilizer. For this reason, we will try to understand the structure of the different stabilizers in the jet schemes $G_{n}$.

Recall from Definition 3.1 that $\mathcal{C}_{\lambda}$ is the orbit containing the following matrix:

$$
\delta_{\lambda}=\left(\begin{array}{ccccccc}
0 & \cdots & 0 & t^{\lambda_{1}} & 0 & \cdots & 0 \\
0 & \cdots & 0 & 0 & t^{\lambda_{2}} & \cdots & 0 \\
\vdots & & \vdots & \vdots & \vdots & \ddots & \vdots \\
0 & \cdots & 0 & 0 & 0 & \cdots & t^{\lambda_{r}}
\end{array}\right)
$$

This matrix defines an element of the jet scheme $\mathcal{M}_{n}$ as long as $n$ is greater than the co-length of $\lambda$; the corresponding $G_{n}$-orbit in $\mathcal{M}_{n}$ is denoted by $\mathcal{C}_{\lambda, n}$. The following proposition determines the codimension of the stabilizer of $\delta_{\lambda}$ in the jet group $G_{n}$.

Proposition 5.3. Let $\lambda \in \Lambda_{r}$ be a partition of length at most $r$, and let $n$ be a positive integer greater than the highest term of $\lambda$. Let $H_{\lambda, n}$ denote the stabilizer of $\delta_{\lambda}$ in the group $G_{n}$. Then

$$
\operatorname{codim}\left(H_{\lambda, n}, G_{n}\right)=(n+1) r s-\sum_{i=1}^{r} \lambda_{i}(s-r+2 i-1) .
$$


Proof. Pick $(g, h) \in G_{n}=\left(\mathrm{GL}_{r}\right)_{n} \times\left(\mathrm{GL}_{s}\right)_{n}$. Then:

$$
\begin{aligned}
(g, h) \in H_{\lambda, n} & \Longleftrightarrow g \cdot \delta_{\lambda} \cdot h^{-1}=\delta_{\lambda} \Longleftrightarrow g \cdot \delta_{\lambda}=\delta_{\lambda} \cdot h \\
& \Longleftrightarrow\left(\begin{array}{ccccccc}
0 & \cdots & 0 & t^{\lambda_{1}} * & t^{\lambda_{2}} * & \cdots & t^{\lambda_{r}} * \\
0 & \cdots & 0 & t^{\lambda_{1}} * & t^{\lambda_{2}} * & \cdots & t^{\lambda_{r}} \\
\vdots & & \vdots & \vdots & \vdots & \ddots & \vdots \\
0 & \cdots & 0 & t^{\lambda_{1}} * & t^{\lambda_{2}} * & \cdots & t^{\lambda_{r}}
\end{array}\right) \\
& =\left(\begin{array}{ccccccc}
t^{\lambda_{1}} * & \cdots & t^{\lambda_{1}} * & t^{\lambda_{1}} * & t^{\lambda_{1}} * & \cdots & t^{\lambda_{1}} * \\
t^{\lambda_{2}} * & \cdots & t^{\lambda_{2}} * & t^{\lambda_{2}} * & t^{\lambda_{2}} * & \cdots & t^{\lambda_{2}} * \\
\vdots & & \vdots & \vdots & \vdots & \ddots & \vdots \\
t^{\lambda_{r}} * & \cdots & t^{\lambda_{r}} * & t^{\lambda_{r}} * & t^{\lambda_{r}} * & \cdots & t^{\lambda_{r}}
\end{array}\right) .
\end{aligned}
$$

This equality of matrices gives one equation of the form $t^{a(i, j)} *=t^{b(i, j)} *$ for each entry $(i, j)$ in an $r \times s$ matrix. We have $a(i, j)=\lambda_{j-s+r}$ and $b(i, j)=\lambda_{i}$ (assume $\lambda_{j}=\infty$ for $\left.j<0\right)$.

Each equation of the form $t^{a} *=t^{b} *$ gives $(n+1)-\min \{a, b\}$ independent equations on the coefficients of the power series, so it reduces the dimension of the stabilizer by $(n+1)-\min \{a, b\}$. The entries $(i, j)$ for which $\min \{a(i, j), b(i, j)\}=\lambda_{k}$ form an $\rightleftharpoons$ shaped region of the $r \times s$ matrix, as we illustrate in the following diagram:

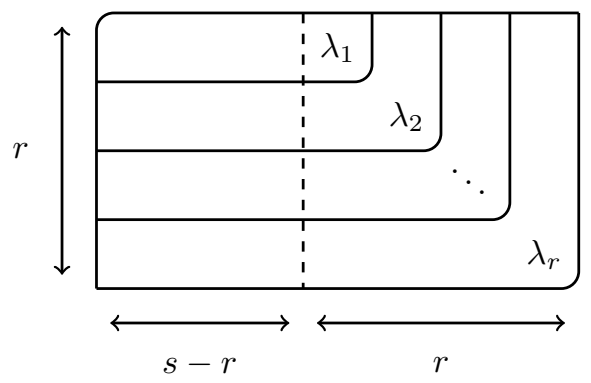

The region corresponding to $\lambda_{i}$ contains $(s-r+2 i-1)$ entries, and the result follows.

Proposition 5.4. Let $\lambda \in \bar{\Lambda}_{r}$ be a pre-partition of length at most $r$, and consider its associated $G_{\infty}$-orbit $\mathcal{C}_{\lambda}$ in $\mathcal{M}_{\infty}$. If $\lambda$ contains infinite terms, $\mathcal{C}_{\lambda}$ has infinite codimension. If $\lambda$ is a partition, the codimension is given by

$$
\operatorname{codim}\left(\mathcal{C}_{\lambda}, \mathcal{M}_{\infty}\right)=\sum_{i=1}^{r} \lambda_{i}(s-r+2 i-1) .
$$

Proof. If $\lambda$ contains infinite terms, $\mathcal{C}_{\lambda}$ is thin, so it has infinite codimension. Otherwise Proposition 3.9 tells us that $\mathcal{C}_{\lambda}$ is the inverse image of $\mathcal{C}_{\lambda, n}$ under the truncation map $\mathcal{M}_{\infty} \rightarrow \mathcal{M}_{n}$ for $n$ large enough. Since $\mathcal{M}$ is smooth, we see that the codimension of $\mathcal{C}_{\lambda}$ in $\mathcal{M}_{\infty}$ is the same as the codimension of $\mathcal{C}_{\lambda, n}$ in $\mathcal{M}_{n}$. The dimension of $\mathcal{C}_{\lambda, n}$ is the codimension of the stabilizer of $\delta_{\lambda}$ in $G_{n}$. The result now follows from Proposition 5.3 and the fact that $\mathcal{M}_{n}$ has dimension $(n+1)$ rs. 
Corollary 5.5. Let $\nu$ be a $G$-invariant divisorial valuation of $\mathcal{M}$, and write $\nu=$ $q \cdot \mathrm{val}_{E}$, where $q$ is a positive integer and $E$ is a divisor in a smooth birational model above $M$. Let $\lambda \in \Lambda_{r}$ be the unique partition such that $\mathcal{C}_{\lambda}$ induces $\nu$, and let $k_{\nu}(\mathcal{M})$ be the discrepancy of $\mathcal{M}$ along $\nu$. Then

$$
k_{\nu}(\mathcal{M})+q=\sum_{i=1}^{r} \lambda_{i}(s-r+2 i-1) .
$$

Proof. From Proposition 5.1 we know that the closure of $\mathcal{C}_{\lambda}$ is the maximal divisorial set associated to $\nu$. Since $\mathcal{M}$ is smooth, the $\log$ discrepancy $k_{\nu}(\mathcal{M})+q$ agrees with the codimension of the associated maximal divisorial set (see Section 1.3). The result now follows from Proposition 5.4.

Theorem 5.6. Recall that $\mathcal{M}$ denotes the space of matrices of size $r \times s$ and $D^{k}$ is the variety of matrices of rank at most $k$. The log canonical threshold of the pair $\left(\mathcal{M}, D^{k}\right)$ is

$$
\operatorname{lct}\left(\mathcal{M}, D^{k}\right)=\min _{i=0, \ldots, k} \frac{(r-i)(s-i)}{k+1-i} .
$$

Proof. We will use Mustaţă's formula (see [ELM04, Cor. 3.2]) to compute log canonical thresholds:

$$
\operatorname{lct}\left(\mathcal{M}, D^{k}\right)=\min _{n}\left\{\frac{\operatorname{codim}\left(D_{n}^{k}, \mathcal{M}_{n}\right)}{n+1}\right\}=\min _{p}\left\{\frac{\operatorname{codim}\left(\operatorname{Cont}^{p}\left(D^{k}\right), \mathcal{M}_{\infty}\right)}{p}\right\} .
$$

Let $\Sigma_{p} \subset \bar{\Lambda}_{r}$ be the set of pre-partitions of length at most $r$ such that $\lambda_{r}+\cdots+$ $\lambda_{r-k}=p$. By Propositions 3.3 and 4.9. we have

$$
\operatorname{lct}\left(\mathcal{M}, D^{k}\right)=\min _{p} \min _{\lambda \in \Sigma_{p}}\left\{\frac{\operatorname{codim}\left(\mathcal{C}_{\lambda}, \mathcal{M}_{\infty}\right)}{p}\right\} .
$$

Consider the following linear function:

$$
\psi\left(a_{1}, \ldots, a_{r}\right)=\sum_{i=1}^{r} a_{i}(s-r+2 i-1) .
$$

Then, by Proposition 5.4 we get

$$
\operatorname{lct}\left(\mathcal{M}, D^{k}\right)=\min _{p} \min _{\lambda \in \Sigma_{p}}\left\{\frac{\psi(\lambda)}{p}\right\}=\min _{p} \min _{\lambda \in \Sigma_{p}}\left\{\psi\left(\frac{\lambda}{p}\right)\right\} .
$$

Let $\Sigma \subset \mathbf{Q}^{r}$ be the set of tuples $\left(a_{1}, \ldots, a_{r}\right)$ such that $a_{1} \geq a_{2} \geq \cdots \geq a_{r} \geq 0$ and $a_{r}+\cdots+a_{r-k}=1$. Then

$$
\operatorname{lct}\left(\mathcal{M}, D^{k}\right)=\min _{a \in \Sigma}\{\psi(a)\} .
$$

The map $\varphi\left(a_{1}, \ldots, a_{r}\right)=\left(a_{1}-a_{2}, \ldots, a_{r-1}-a_{r}, a_{r}\right)$ sends $\Sigma$ to $\Sigma^{\prime}$, where $\Sigma^{\prime} \subset \mathbf{Q}^{r}$ is the set of tuples $\left(b_{1}, \ldots, b_{r}\right)$ such that $b_{i} \geq 0$ and $(k+1) b_{r}+k b_{r-1}+\cdots+b_{r-k}=1$. Then

$$
\operatorname{lct}\left(\mathcal{M}, D^{k}\right)=\min _{b \in \Sigma^{\prime}}\{\xi(b)\},
$$

where

$$
\xi(b)=\psi\left(\varphi^{-1}(b)\right)=\sum_{i=1}^{r}\left(b_{r}+b_{r-1}+\cdots+b_{i}\right)(s-r+2 i-1)=\sum_{j=1}^{r} b_{j} j(s-r+j) .
$$

Note that in the definition of $\Sigma^{\prime}$ the only restriction on the first $r-k-1$ coordinates $b_{1}, b_{2}, \ldots, b_{r-k-1}$ is that they are non-negative. Let $\Sigma^{\prime \prime}$ be the subset of $\Sigma^{\prime}$ obtained 
by setting $b_{1}=\cdots=b_{r-k-1}=0$. From the formula for $\xi(b)$ we see that the minimum $\min _{b \in \Sigma^{\prime}}\{\xi(b)\}$ must be achieved in $\Sigma^{\prime \prime}$. But $\Sigma^{\prime \prime}$ is a simplex and $\xi$ is linear, so the minimum is actually achieved in one of the extremal points of $\Sigma^{\prime \prime}$. These extremal points are:

$$
\begin{gathered}
P_{r-k}=(0, \ldots, 0,1,0, \ldots, 0,0), \quad P_{r-k+1}=\left(0, \ldots, 0,0, \frac{1}{2}, \ldots, 0,0\right), \quad \ldots, \\
P_{r-1}=\left(0, \ldots, 0,0,0, \ldots, \frac{1}{k}, 0\right), \quad P_{r}=\left(0, \ldots, 0,0,0, \ldots, 0, \frac{1}{k+1}\right) .
\end{gathered}
$$

The value of $\xi$ at these points is

$$
\xi\left(P_{r-i}\right)=\frac{1}{k+1-i}(r-i)(s-i) .
$$

Therefore

$$
\operatorname{lct}\left(\mathcal{M}, D^{k}\right)=\min _{i=0, \ldots, k} \frac{(r-i)(s-i)}{k+1-i}
$$

as required.

\section{Motivic INTEGRATion}

In the previous section we computed codimensions of orbits in the arc space $\mathcal{M}_{\infty}$ as a means to obtain formulas for discrepancies and log canonical thresholds. But a careful look at the proofs shows that we can understand more about the orbits than just their codimensions. As an example of this, in this section we compute the motivic volume of the orbits in the arc space. This allows us to determine topological zeta functions of determinantal varieties.

Throughout this section, we will restrict ourselves to the case of square matrices, i.e. we assume $r=s$.

6.1. Motivic volume of orbits. Before we state the main proposition, we need to recall some notions from the group theory of $\mathrm{GL}_{r}$ : parabolic subgroups, Levi factors, flag manifolds, and the natural way to obtain a parabolic subgroup from a partition.

Definition 6.1. Let $0<v_{1}<v_{2}<\cdots<v_{j}<r$ be integers. A flag in $\mathbf{C}^{r}$ of signature $\left(v_{1}, \ldots, v_{j}\right)$ is a nested chain $V_{1} \subset V_{2} \subset \cdots \subset V_{j} \subset \mathbf{C}^{r}$ of vector subspaces with $\operatorname{dim} V_{i}=v_{i}$. The general linear group $\mathrm{GL}_{r}$ acts transitively on the set of all flags with a given signature. The stabilizer of a flag is known as a parabolic subgroup of $\mathrm{GL}_{r}$. If $P \subset \mathrm{GL}_{r}$ is a parabolic subgroup, the quotient $\mathrm{GL}_{r} / P$ parametrizes flags of a given signature and is known as a flag variety.

Definition 6.2. Let $\left\{e_{1}, \ldots, e_{r}\right\}$ be the standard basis for $\mathbf{C}^{r}$, and let

$$
\lambda=\left(d_{1}^{a_{1}} \ldots d_{j}^{a_{j}}\right) \in \Lambda_{r}
$$

be a partition. Write $a_{j+1}=r-\sum_{i=1}^{j} a_{i}$ and $v_{i}=a_{1}+\cdots+a_{i}$, and consider the following vector subspaces of $\mathbf{C}^{r}$ :

$$
V_{i}=\operatorname{span}\left(e_{1}, \ldots, e_{v_{i}}\right), \quad W_{i}=\operatorname{span}\left(e_{v_{i-1}+1}, \ldots, e_{v_{i}}\right) .
$$

We denote by $P_{\lambda}$ the stabilizer of the flag $V_{1} \subset \cdots \subset V_{j}$ and call it the parabolic subgroup of $\mathrm{GL}_{r}$ associated to $\lambda$. The group $L_{\lambda}=\mathrm{GL}_{a_{1}} \times \cdots \times \mathrm{GL}_{a_{j+1}}$ embeds naturally in $P_{\lambda}$ as the group endomorphisms of $W_{i}$, and is known as the Levi factor of the parabolic $P_{\lambda}$. 
Example 6.3. Assume $r=6$ and consider the partition $\lambda=(4,4,4,1,1)=\left(4^{3} 1^{2}\right)$. Then $P_{\lambda}$ and $L_{\lambda}$ are the groups of invertible $r \times r$ matrices of the forms

$$
P_{\lambda}:\left(\begin{array}{cccccc}
* & * & * & * & * & * \\
* & * & * & * & * & * \\
* & * & * & * & * & * \\
0 & 0 & 0 & * & * & * \\
0 & 0 & 0 & * & * & * \\
0 & 0 & 0 & 0 & 0 & *
\end{array}\right), \quad L_{\lambda}:\left(\begin{array}{cccccc}
* & * & * & 0 & 0 & 0 \\
* & * & * & 0 & 0 & 0 \\
* & * & * & 0 & 0 & 0 \\
0 & 0 & 0 & * & * & 0 \\
0 & 0 & 0 & * & * & 0 \\
0 & 0 & 0 & 0 & 0 & *
\end{array}\right)
$$

Proposition 6.4. Assume that $r=s$. Let $\lambda \in \Lambda_{r}$ be a partition of length at most $r$ and consider its associated parabolic subgroup $P_{\lambda}$ and Levi factor $L_{\lambda}$. Let $\mu$ be the motivic measure in $\mathcal{M}_{\infty}$, and $\mathcal{C}_{\lambda}$ the orbit in $\mathcal{M}_{\infty}$ associated to $\lambda$. If $b$ is the log discrepancy of the valuation induced by $\mathcal{C}_{\lambda}$, we have

$$
\mu\left(\mathcal{C}_{\lambda}\right)=\mathbf{L}^{-b}\left[\mathrm{GL}_{r} / P_{\lambda}\right]^{2}\left[L_{\lambda}\right]
$$

Proof. Consider $n, \delta_{\lambda}$ and $H_{\lambda, n} \subset G_{n}$ as in Proposition 5.3. If $\mathcal{C}_{\lambda, n}$ is the truncation of $\mathcal{C}_{\lambda}$ to $\mathcal{M}_{n}$, we know that for $n$ large enough

$$
\mu\left(\mathcal{C}_{\lambda}\right)=\mathbf{L}^{-r^{2} n}\left[\mathcal{C}_{\lambda, n}\right]=\mathbf{L}^{-r^{2} n}\left[G_{n}\right]\left[H_{\lambda, n}\right]^{-1}=\mathbf{L}^{r^{2} n}\left[\mathrm{GL}_{r}\right]^{2}\left[H_{\lambda, n}\right]^{-1} .
$$

At the beginning of the proof of Proposition 5.3 we found the equations defining $H_{\lambda, n}$ :

$$
(g, h) \in H_{\lambda, n} \quad \Leftrightarrow \quad g \cdot \delta_{\lambda} \cdot h^{-1}=\delta_{\lambda} \quad \Leftrightarrow \quad g \cdot \delta_{\lambda}=\delta_{\lambda} \cdot h \quad \Leftrightarrow
$$

$$
\left(\begin{array}{cccc}
t^{\lambda_{1}} * & t^{\lambda_{2}} * & \cdots & t^{\lambda_{r}} * \\
t^{\lambda_{1}} * & t^{\lambda_{2}} * & \cdots & t^{\lambda_{r}} * \\
\vdots & \vdots & \ddots & \vdots \\
t^{\lambda_{1}} * & t^{\lambda_{2}} * & \cdots & t^{\lambda_{r}}
\end{array}\right)=\left(\begin{array}{cccc}
t^{\lambda_{1}} * & t^{\lambda_{1}} * & \cdots & t^{\lambda_{1}} * \\
t^{\lambda_{2}} * & t^{\lambda_{2}} * & \cdots & t^{\lambda_{2}} * \\
\vdots & \vdots & \ddots & \vdots \\
t^{\lambda_{r}} * & t^{\lambda_{r}} & \cdots & t^{\lambda_{r}}
\end{array}\right) .
$$

As a variety, $G_{n}$ can be written as product $G \times \mathfrak{g}^{2 n}$, where $\mathfrak{g} \simeq \mathbf{A}^{r^{2}}$ is the Lie algebra of $\mathrm{GL}_{r}$. Let $g_{i, j}^{(k)}$ and $h_{i, j}^{(k)}$ be the natural coordinates on $G_{n}=\left(\mathrm{GL}_{r} \times \mathfrak{g}\right)^{2}$, where $g_{i, j}^{(0)}=g_{i, j}$ and $h_{i, j}^{(0)}=h_{i, j}$ are coordinates for $G=\mathrm{GL}_{r} \times \mathrm{GL}_{r}$. Then, for $n$ large enough, the equations in (11) can be expressed as

$$
t^{\lambda_{j}} \sum_{k=0}^{n} g_{i, j}^{(k)} t^{k}=t^{\lambda_{i}} \sum_{k=0}^{n} h_{i, j}^{(k)} t^{k} \quad \bmod t^{n+1} .
$$

Let $H \subset G$ be the truncation of $H_{\lambda, n}$. Then $H$ is the subgroup of $G$ given by those equations in (2) involving only the variables $g_{i, j}$ and $h_{i, j}$; these equations are

$$
\begin{array}{ll}
g_{i, j}=h_{i, j} & \text { if } \lambda_{i}=\lambda_{j}, \\
g_{i, j}=0 & \text { if } \lambda_{i} \neq \lambda_{j} \text { and } i<j, \\
h_{i, j}=0 & \text { if } \lambda_{i} \neq \lambda_{j} \text { and } i>j .
\end{array}
$$

From (41) and (5), we see that $H$ is a subgroup of $P_{\lambda}^{\mathrm{op}} \times P_{\lambda} \subset G$, and (3) tells us that we can obtain $H$ from $P_{\lambda}^{\mathrm{op}} \times P_{\lambda}$ by identifying the two copies of the Levi $L_{\lambda}$. Hence $[H]=\left[P_{\lambda}\right]^{2}\left[L_{\lambda}\right]^{-1}$.

From (2) we also see that $H_{\lambda, n}$ is a sub-bundle of $H \times \mathfrak{g}^{2 n}$. More precisely, if $\mathfrak{h}$ is the fiber of $H_{\lambda, n}$ over the identity in $H$, then $\mathfrak{h} \subset \mathfrak{g}^{2 n}$ is an affine space and all the 
fibers of $H_{\lambda, n}$ are isomorphic to $\mathfrak{h}$. The codimension of $\mathfrak{h}$ in $\mathfrak{g}^{2 n}$ can be computed with the same method used in the proof of Proposition 5.3 ,

$$
\operatorname{codim}\left(\mathfrak{h}, \mathfrak{g}^{2 n}\right)=n r^{2}-\sum_{i=1}^{r} \lambda_{i}(2 i-1)=n r^{2}-b
$$

where $b$ is the $\log$ discrepancy of the valuation induced by $\mathcal{C}_{\lambda}$. As a consequence,

$$
[\mathfrak{h}]=\left[\mathfrak{g}^{2 n}\right] \mathbf{L}^{-n r^{2}+b}=\mathbf{L}^{n r^{2}+b}
$$

and

$$
\begin{aligned}
\mu\left(\mathcal{C}_{\lambda}\right) & =\mathbf{L}^{r^{2} n}\left[\mathrm{GL}_{r}\right]^{2}\left[H_{\lambda, n}\right]^{-1}=\mathbf{L}^{r^{2} n}\left[\mathrm{GL}_{r}\right]^{2}[H]^{-1}[\mathfrak{h}]^{-1}=\mathbf{L}^{-b}\left[\mathrm{GL}_{r}\right]^{2}[H]^{-1} \\
& =\mathbf{L}^{-b}\left[\mathrm{GL}_{r}\right]^{2}\left[P_{\lambda}\right]^{-2}\left[L_{\lambda}\right]=\mathbf{L}^{-b}\left[\mathrm{GL}_{r} / P_{\lambda}\right]^{2}\left[L_{\lambda}\right] .
\end{aligned}
$$

6.2. Topological zeta function. Recall from Section 1.4 that the motivic Igusa zeta function for the pair $\left(\mathcal{M}, D^{k}\right)$ is defined as

$$
Z_{D^{k}}(s):=\int_{\mathcal{M}_{\infty}}\left|\mathcal{I}_{D^{k}}\right|^{s} d \mu=\sum_{p=0}^{\infty} \mu\left(\text { Cont }^{=p} D^{k}\right) \mathbf{L}^{-s p}
$$

where $\mu$ is the motivic measure on $\mathcal{M}_{\infty}$ and $\mathbf{L}^{-s}$ is considered as a formal variable. The topological zeta function $Z_{D^{k}}^{\text {top }}(s)$ can be obtained from the Igusa zeta function by formally expanding $Z_{D^{k}}(s)$ as a power series in $(\mathbf{L}-1)$ and then extracting the constant term (i.e. by specializing $\mathbf{L}$ to $\left.\chi\left(\mathbf{A}^{1}\right)=1\right)$.

Using Propositions 3.3 and 6.4 we can write $Z_{D^{k}}(s)$ as (6)

$$
Z_{D^{k}}(s)=\sum_{\lambda \in \Lambda_{r}} \mu\left(\mathcal{C}_{\lambda}\right) \mathbf{L}^{-s\left(\lambda_{r}+\cdots+\lambda_{r-k}\right)}=\sum_{\lambda \in \Lambda_{r}}\left[\mathrm{GL}_{r} / P_{\lambda}\right]^{2}\left[L_{\lambda}\right] \mathbf{L}^{-b_{\lambda}-s\left(\lambda_{r}+\cdots+\lambda_{r-k}\right)},
$$

where $b_{\lambda}=\sum_{i=1}^{r} \lambda_{i}(2 i-1)$ is the $\log$ discrepancy of the valuation induced by $\mathcal{C}_{\lambda}$. There are only finitely many possibilities for the value of $\left[\mathrm{GL}_{r} / P_{\lambda}\right]^{2}\left[L_{\lambda}\right]$, and it will be convenient to group the terms in the sum above accordingly. In order to do so, consider the bijection between $\Lambda_{r}$ and $\mathbf{N}^{r}$ given by

$$
\begin{gathered}
\lambda=\left(\lambda_{1}, \lambda_{2}, \ldots, \lambda_{r}\right) \in \Lambda_{r} \quad \longmapsto \quad a(\lambda)=\left(\lambda_{1}-\lambda_{2}, \lambda_{2}-\lambda_{3}, \ldots, \lambda_{r}\right) \in \mathbf{N}^{r}, \\
a=\left(a_{1}, a_{2}, \ldots, a_{r}\right) \in \mathbf{N}^{r} \longmapsto \lambda(a)=\left(a_{1}+\cdots+a_{r}, a_{2}+\cdots+a_{r}, \ldots, a_{r}\right) \in \Lambda_{r} .
\end{gathered}
$$

For a subset $I \subseteq\{1, \ldots, r-1\}$, consider $I^{c}=\{1, \ldots, r-1\} \backslash I$ and define

$$
\Omega_{I}=\left\{a \in \mathbf{N}^{r}:\left(a_{i}=0 \forall i \in I\right) \text { and }\left(a_{j} \neq 0 \forall j \in I^{c}\right)\right\} .
$$

Let $\lambda$ and $\lambda^{\prime}$ be two partitions such that both $a(\lambda)$ and $a\left(\lambda^{\prime}\right)$ belong to $\Omega_{I}$ for some $I$. From the definitions of $P_{\lambda}$ and $L_{\lambda}$ (see Section 6.1) we see that $\left[\mathrm{GL}_{r} / P_{\lambda}\right]^{2}\left[L_{\lambda}\right]=$ $\left[\mathrm{GL}_{r} / P_{\lambda^{\prime}}\right]^{2}\left[L_{\lambda^{\prime}}\right]$. Hence, given a subset $I \subseteq\{1, \ldots, r-1\}$ we can consider $\eta(I)=$ $\left[\mathrm{GL}_{r} / P_{\lambda}\right]^{2}\left[L_{\lambda}\right]$, where $\lambda$ is any partition with $a(\lambda) \in \Omega_{I}$, and we obtain a welldefined function on the subsets of $\{1, \ldots, r-1\}$.

Fix a subset $I \subseteq\{1, \ldots, r-1\}$ and a partition $\lambda$ such that $a(\lambda) \in \Omega_{I}$. Consider $I_{r}^{c}=\{1, \ldots, r\} \backslash I=\left\{i_{1}, \ldots, i_{\ell}\right\}$, where $i_{j}<i_{j+1}$. Set $i_{0}=0$. Then $\mathrm{GL}_{r} / P_{\lambda}$ is the manifold of partial flags of signature $\left(i_{1}, \ldots, i_{\ell}\right)$, and its class in the Grothendieck group of varieties is given by

$$
\left[\mathrm{GL}_{r} / P_{\lambda}\right]=\prod_{j=1}^{\ell}\left[G\left(i_{j-1}, i_{j}\right)\right]=\prod_{j=1}^{\ell}\left[G\left(i_{j}-i_{j-1}, i_{j}\right)\right]
$$


where $G(u, v)$ is the Grassmannian of $u$-dimensional vector subspaces of $\mathbf{C}^{v}$. Analogously,

$$
\left[L_{\lambda}\right]=\prod_{j=1}^{\ell}\left[\mathrm{GL}_{i_{j}-i_{j-1}}\right] .
$$

If we define $d\left(I, i_{j}\right)=i_{j}-i_{j-1}$ for $i_{j} \in I_{r}^{c}$, we can write

$$
\eta(I)=\left[\mathrm{GL}_{r} / P_{\lambda}\right]^{2}\left[L_{\lambda}\right]=\prod_{i \notin I}[G(d(I, i), i)]^{2}\left[\mathrm{GL}_{d(I, i)}\right] .
$$

This shows more explicitly that $\eta(I)$ depends only on $I$, and not on the particular partition $\lambda$ in $\Omega_{I}$. From equation (6) we obtain

$$
Z_{D^{k}}(s)=\sum_{I \subseteq\{1, \ldots, r-1\}}\left(\eta(I) \sum_{a(\lambda) \in \Omega_{I}} \mathbf{L}^{-b_{\lambda}-s\left(\lambda_{r}+\cdots+\lambda_{r-k}\right)}\right) .
$$

Consider

$$
\psi(a)=b_{\lambda(a)}+s\left(\lambda(a)_{r}+\cdots+\lambda(a)_{r-k}\right)=\psi_{1} a_{1}+\cdots+\psi_{r} a_{r},
$$

where

$$
\psi_{i}=i^{2}+s \max \{0, k+1+i-r\}
$$

Then

$$
\begin{aligned}
& \sum_{a(\lambda) \in \Omega_{I}} \mathbf{L}^{-b_{\lambda}-s\left(\lambda_{r}+\cdots+\lambda_{r-k}\right)} \\
& =\sum_{a \in \Omega_{I}} \mathbf{L}^{-\psi_{1} a_{1}-\cdots-\psi_{r} a_{r}}=\sum_{a \in \Omega_{I}} \mathbf{L}^{-\sum_{i \notin I} \psi_{i} a_{i}} \\
& =\left(\prod_{\substack{i \notin I \\
i \neq r}} \sum_{a_{i}=1}^{\infty} \mathbf{L}^{-\psi_{i} a_{i}}\right) \cdot\left(\sum_{a_{r}=0}^{\infty} \mathbf{L}^{-\psi_{r} a_{r}}\right) \\
& =\mathbf{L}^{\psi_{r}} \cdot \prod_{i \notin I} \frac{\mathbf{L}^{-\psi_{i}}}{1-\mathbf{L}^{-\psi_{i}}}=\mathbf{L}^{\psi_{r}} \cdot \prod_{i \notin I} \frac{1}{\mathbf{L}^{\psi_{i}-1}} .
\end{aligned}
$$

Combining equations (7), (8), and (10), we get

$$
Z_{D^{k}}(s)=\mathbf{L}^{\psi_{r}} \sum_{I \subseteq\{1, \ldots, r-1\}} \prod_{i \notin I} \frac{1}{\mathbf{L}^{\psi_{i}}-1}[G(d(I, i), i)]^{2}\left[\mathrm{GL}_{d(I, i)}\right] .
$$

We will not try to simplify equation (11) any further. Instead, we will use it to compute the topological zeta function. As explained in Section 1.4 it is enough to expand each summand in (11) as a power series in $(\mathbf{L}-1)$. We will write $O\left((\mathbf{L}-1)^{\ell}\right)$ to denote a power series in $(\mathbf{L}-1)$ which is divisible by $(\mathbf{L}-1)^{\ell}$. We have

$$
\mathbf{L}^{\psi_{i}}=1+O(\mathbf{L}-1), \quad \mathbf{L}^{\psi_{i}}-1=\psi_{i} \cdot(\mathbf{L}-1)+O\left((\mathbf{L}-1)^{2}\right),
$$

and

$$
[G(1, i)]=\left[\mathbf{P}^{i-1}\right]=1+\mathbf{L}+\mathbf{L}^{2}+\cdots+\mathbf{L}^{i-1}=i+O(\mathbf{L}-1) .
$$


The class of $\mathrm{GL}_{d}$ can be computed by induction on $d$. Let $P=P_{(1)}$ be the parabolic subgroup of $\mathrm{GL}_{d}$ whose corresponding quotient is projective space $\mathrm{GL}_{d} / P=\mathbf{P}^{d-1}$. Then, for $d>1$,

$$
[P]=\left[\mathrm{GL}_{1}\right]\left[\mathrm{GL}_{d-1}\right] \mathbf{L}^{d-1}=\left[\mathrm{GL}_{1}\right]\left[\mathrm{GL}_{d-1}\right](1+O(\mathbf{L}-1)), \quad\left[\mathbf{P}^{d-1}\right]=d+O(\mathbf{L}-1) .
$$

Since $\left[\mathrm{GL}_{1}\right]=\mathbf{L}-1$, we see that

$$
\left[\mathrm{GL}_{d}\right]=[P]\left[\mathbf{P}^{d-1}\right]=\left[\mathrm{GL}_{1}\right]\left[\mathrm{GL}_{d-1}\right](d+O(\mathbf{L}-1))=O\left((\mathbf{L}-1)^{d}\right) .
$$

Hence

$$
\frac{1}{\mathbf{L}^{\psi_{i}}-1}[G(d, i)]^{2}\left[\mathrm{GL}_{d}\right]=O\left((\mathbf{L}-1)^{d-1}\right)
$$

and

$$
\frac{1}{\mathbf{L}^{\psi_{i}}-1}[G(1, i)]^{2}\left[\mathrm{GL}_{1}\right]=\frac{i^{2}}{\psi_{i}}+O(\mathbf{L}-1) .
$$

In particular, the only summands in equation (11) not divisible by $(\mathbf{L}-1)$ are those for which $d(I, i)=1$ for all $i \notin I$. Since $d(I, i)=1$ if and only if $i-1 \notin I$, the only significant summand is the one corresponding to $I=\emptyset$. Hence

$$
Z_{D^{k}}(s)=\prod_{i=1}^{r} \frac{i^{2}}{\psi_{i}}+O(\mathbf{L}-1) .
$$

Combining this with equation (9) we get the topological zeta function

$$
\begin{aligned}
Z_{D^{k}}^{\mathrm{top}}(s) & =\prod_{i=1}^{r} \frac{i^{2}}{\psi_{i}}=\prod_{i=1}^{r-k-1} \frac{i^{2}}{i^{2}} \prod_{i=r-k}^{r} \frac{i^{2}}{i^{2}+s(k+1-i-r)} \\
& =\prod_{i=r-k}^{r}\left(1+s \frac{k+1-i-r}{i^{2}}\right)^{-1}=\prod_{j=0}^{k}\left(1+s \frac{k+1-j}{(r-j)^{2}}\right)^{-1} .
\end{aligned}
$$

The following theorem summarizes the results of this section.

Theorem 6.5. Let $\mathcal{M}=\mathbf{A}^{r^{2}}$ be the space of square $r \times r$ matrices, and let $D^{k}$ be the subvariety of matrices of rank at most $k$. Then the topological zeta function of the pair $\left(\mathcal{M}, D^{k}\right)$ is given by

$$
Z_{D^{k}}^{\mathrm{top}}(s)=\prod_{\zeta \in \Omega} \frac{1}{1-s \zeta^{-1}}
$$

where $\Omega$ is the set of poles

$$
\Omega=\left\{-\frac{r^{2}}{k+1}, \quad-\frac{(r-1)^{2}}{k}, \quad-\frac{(r-2)^{2}}{k-1}, \quad \ldots, \quad-(r-k)^{2}\right\} .
$$

\section{ACKNOWLEDGMENTS}

The author is very grateful to his thesis advisor, Lawrence Ein, for the introduction to the world of arc spaces and for suggesting this problem. His guidance and support during the author's years as a graduate student made this work possible. The author would also like to thank Karen Smith and Mircea Mustaţă for their valuable comments and suggestions on an earlier version of this paper. 


\section{REFERENCES}

[BV88] Winfried Bruns and Udo Vetter, Determinantal rings, Lecture Notes in Mathematics, vol. 1327, Springer-Verlag, Berlin, 1988. MR953963 (89i:13001)

[dCEP80] C. de Concini, David Eisenbud, and C. Procesi, Young diagrams and determinantal varieties, Invent. Math. 56 (1980), no. 2, 129-165. MR.558865(81m:14034)

[dFEI08] Tommaso de Fernex, Lawrence Ein, and Shihoko Ishii, Divisorial valuations via arcs, Publ. Res. Inst. Math. Sci. 44 (2008), no. 2, 425-448. MR2426354 (2010d:14055)

[DL98] Jan Denef and François Loeser, Motivic Igusa zeta functions, J. Algebraic Geom. 7 (1998), no. 3, 505-537. MR1618144 (99j:14021)

[DL99] Germs of arcs on singular algebraic varieties and motivic integration, Invent. Math. 135 (1999), no. 1, 201-232. MR1664700 (99k:14002)

[DL02] Motivic integration, quotient singularities and the McKay correspondence, Compositio Math. 131 (2002), no. 3, 267-290. MR1905024(2004e:14010)

[ELM04] Lawrence Ein, Robert Lazarsfeld, and Mircea Mustaţă, Contact loci in arc spaces, Compos. Math. 140 (2004), no. 5, 1229-1244. MR2081163 (2005f:14006)

[EM06] Lawrence Ein and Mircea Mustaţă, Invariants of singularities of pairs, International Congress of Mathematicians. Vol. II, Eur. Math. Soc., Zürich, 2006, pp. 583-602. MR2275611 (2007m:14050)

[EMY03] Lawrence Ein, Mircea Mustaţă, and Takehiko Yasuda, Jet schemes, log discrepancies and inversion of adjunction, Invent. Math. 153 (2003), no. 3, 519-535. MR2000468 (2004f:14028)

[Ful97] William Fulton, Young tableaux. With applications to representation theory and geometry. London Mathematical Society Student Texts, vol. 35, Cambridge University Press, Cambridge, 1997. MR1464693 (99f:05119)

[GS06] Russell A. Goward, Jr. and Karen E. Smith, The jet scheme of a monomial scheme, Comm. Algebra 34 (2006), no. 5, 1591-1598. MR2229478 (2007e:13037)

[Ish04] Shihoko Ishii, The arc space of a toric variety, J. Algebra 278 (2004), no. 2, 666-683. MR2071659 (2005f:14100)

[Ish08] - Maximal divisorial sets in arc spaces, Algebraic geometry in East Asia-Hanoi 2005, Adv. Stud. Pure Math., vol. 50, Math. Soc. Japan, Tokyo, 2008, pp. 237-249. MR2409559 (2009k:14003)

[Joh03] Amanda Ann Johnson, Multiplier ideals of determinantal ideals, Ph.D. thesis, University of Michigan, 2003. MR 2704808

[Kon95] Maxim Kontsevich, Motivic integration, 1995, Lecture at Orsay.

[KS05a] Tomaž Košir and B. A. Sethuraman, Determinantal varieties over truncated polynomial rings, J. Pure Appl. Algebra 195 (2005), no. 1, 75-95. MR.2100311 (2005h:13020)

[KS05b] $\quad$ A Groebner basis for the $2 \times 2$ determinantal ideal $\bmod t^{2}$, J. Algebra 292 (2005), no. 1, 138-153. MR2166800 (2006f:13011)

[Lak87] Dan Laksov, Completed quadrics and linear maps, Algebraic geometry, Bowdoin, 1985 (Brunswick, Maine, 1985), Proc. Sympos. Pure Math., vol. 46, Amer. Math. Soc., Providence, RI, 1987, pp. 371-387. MR.927988 (89c:14077)

[LJ90] Monique Lejeune-Jalabert, Courbes tracées sur un germe d'hypersurface, American Journal of Mathematics 112 (1990), no. 4, 525-568. MR1064990 (92a:14002)

[LJR98] Monique Lejeune-Jalabert and Ana Reguera, Arcs and wedges on sandwiched surface singularities, C. R. Acad. Sci. Paris Sér. I Math. 326 (1998), no. 2, 207-212. MR.1646920 (99g:14045)

[LJR08] Exceptional divisors which are not uniruled belong to the image of the nash map, Arxiv preprint arXiv:0811.2421 (2008).

[Mus01] Mircea Mustaţă, Jet schemes of locally complete intersection canonical singularities, Invent. Math. 145 (2001), no. 3, 397-424. With an appendix by David Eisenbud and Edward Frenkel. MR.1856396 (2002f:14005)

[Mus02] _ Singularities of pairs via jet schemes, J. Amer. Math. Soc. 15 (2002), no. 3, 599-615 (electronic). MR1896234 (2003b:14005)

[Nas95] John F. Nash, Jr., Arc structure of singularities, Duke Math. J. 81 (1995), no. 1, 31-38 (1996). A celebration of John F. Nash, Jr. MR1381967 (98f:14011)

[Nob91] Augusto Nobile, On Nash theory of arc structure of singularities, Ann. Mat. Pura Appl. (4) 160 (1991), 129-146 (1992). MR 1163204 (93h:32047) 
[Plé05] Camille Plénat, Résolution du problème des arcs de Nash pour les points doubles rationnels $D_{n}$, C. R. Math. Acad. Sci. Paris 340 (2005), no. 10, 747-750. MR.2141063 (2005m:14020)

[PPP06] Camille Plénat and Patrick Popescu-Pampu, A class of non-rational surface singularities with bijective Nash map, Bull. Soc. Math. France 134 (2006), no. 3, 383-394. MR.2245998 (2007f:32037)

[Sem51] John G. Semple, The variety whose points represent complete collineations of $S_{r}$ on $S_{r}^{\prime}$, Univ. Roma. Ist. Naz. Alta Mat. Rend. Mat. e Appl. (5) 10 (1951), 201-208. MR.0048847 (14:78c)

[Tyr56] J. A. Tyrrell, Complete quadrics and collineations in $S_{n}$, Mathematika 3 (1956), 69-79. MR0080352(18:233e)

[Vai84] Israel Vainsencher, Complete collineations and blowing up determinantal ideals, Math. Ann. 267 (1984), no. 3, 417-432. MR738261 (85f:14053)

[Vey06] Willem Veys, Arc spaces, motivic integration and stringy invariants, Singularity theory and its applications, Adv. Stud. Pure Math., vol. 43, Math. Soc. Japan, Tokyo, 2006, pp. 529-572. MR2325153 (2008g:14023)

[Voj07] Paul Vojta, Jets via Hasse-Schmidt derivations, Diophantine geometry, CRM Series, vol. 4, Ed. Norm., Pisa, 2007, pp. 335-361. MR2349665 (2008i:13043)

[Yue07a] Cornelia Yuen, Jet schemes of determinantal varieties, Algebra, geometry and their interactions, Contemp. Math., vol. 448, Amer. Math. Soc., Providence, RI, 2007, pp. 261270. MR 2389248 (2009b:14093)

[Yue7b] - The multiplicity of jet schemes of a simple normal crossing divisor, Comm. Algebra 35 (2007), no. 12, 3909-3911. MR2372306 (2008i:13051)

Department of Mathematics, University of Utah, 155 S 1400 E, Room 233, Salt Lake City, UTAн 84112

E-mail address: docampo@math.utah.edu

URL: http://www.math.utah.edu/ docampo 